\title{
Phylogénèse et Systématique Le Phylum des Pentastomida
}

\author{
par R.-M. NICOLI
}

«Les Pentastomides sont aux Arthropodes
ce que sont les Cestodes aux Plathelminthes,
un clade hautement spécialisé. »

Il n'existe guère de groupes zoologiques aussi énigmatiques que les Pentastomides. Aussi, les premiers naturalistes n'ont-ils pas su où les classer et on a vu tour à tour ces êtres dans les Cestodes, les Acanthocéphales, les Hirudinées, les Nématodes, les Crustacés, les Arachnides... Ce sont ces multiples hésitations, avancées et reculs, que nous croyons utile tout d'abord de relever. Puis, dans un second chapitre, nous résumerons les connaissances systématiques actuelles sur ce groupe en établissant un certain nombre de tableaux synoptiques. Enfin, nous évoquerons, en achevant, le problème phylogénétique lui-même et tenterons une synthèse originale.

\section{Historique}

Il semble bien que les Pentastomides soient connus depuis longtemps. Avant J. A. Frölich (1789), traditionnellement regardé comme le premier descripteur d'un Linguatulide, d'autres auteurs avaient signalé ces parasites, tel Wrisberg, 1765, Chabert, 1767 qui décrivit un « ver » du sinus frontal du cheval sous le nom de Ténia lancéolé, que nous rapportons sans peine au genre Linguatula.

En 1789, simultanément, deux auteurs signalent le même parasite sous sa forme larvaire : J. A. Frölich dans le poumon d'un lièvre sous le nom qui restera de Linguatula serrata, Abildgaard à la surface du foie d'un bouc au Danemark sous le nom de Taenia caprina et, en 1789 également, Meyer décrit la forme adulte de la même espèce (Taenia rhinaria).

En 1799, A. de Humboldt fait connaître un parasite d'un serpent, le crotale à sonnette américain (Crotalus adamanteus Palisot de Beauvois, $1799=$ Crotalus durissus G.-A. Boulenger, 1896). C'est l'Echinorhynchus crotali que quelques pages plus loin, 
dans le même ouvrage, il considère comme un Distome Distoma crotali et pour lequel il fondera en 1809 le genre Porocephalus.

En 1800, Gmelin reprend le nom de Taenia caprina. En 1802, Pilger redécrit l'adulte de Linguatula serrata sous le nom de Taenia rhinaria. Puis, en 1803, Zeder distingue abusivement deux genres, le genre Halysis pour Taenia caprina et Linguatula serrata et le genre Polystoma pour Taenia rhinaria. En 1805, Rudolphi rapporte les espèces connues au genre Cochlus bien vite abandonné. Evidemment, les naturalistes de l'époque ignoraient totalement la nature d'un cycle parasitaire (1). Cette confusion, en 1808-1809, Rudolphi la maintient en créant le genre Prionoderma pour le Ténia lancéolé et dans la même publication, un peu plus loin, le genre Polystoma pour le Taenia rhinaria (Polystoma taenioüdes).

En 1811, Bosc décrit sous le nom de Tetragalus caviae un Pentastomide trouvé par Le Gallois dans le poumon d'un cochon d'Inde. C'est cette même espèce que Rudolphi en 1819 désignera sous le nom de Pentastoma emarginatum (il s'agit en fait de la forme larvaire du Linguatula serrata).

En 1816, J.-B. de Lamarck reprend le genre Linguatula et, en 1817, G. Cuvier le genre Prionoderma (Prionoderma lanceolata). En 1819, Rudolphi crée le genre Pentastoma (Pentastome) qui allait donner son nom au groupe entier.

En 1824, H.-D. de Blainville distingue le genre Nettorhynque que J.C. Zenker latinise en Nettorhynchus (2). En 1829, Creplin décrit sous le nom de Pentastoma fera un parasite du foie du chat domestique. C'est encore une forme larvaire de Linguatula serrata.

En 1830, G. Cuvier regroupe toutes les espèces connues dans le genre Linguatula et les place dans son ordre des «Intestinaux cavitaires 》 au voisinage des « Nématoïdes ».

En 1835, Owen reprend dans le même sens le genre Linguatula et considère ces êtres comme des vers hermaphrodites, ce qui constitue une erreur très gênante.

Cette même année (1835), Diesing fait paraître la première monographie du groupe et reconnaît onze espèces différentes dans la section des Acanthotheca.

(1) Remarquons cependant que, dès 1781, Abildgaard avait établi que le canard pouvait être l'hôte définitif du Schistocéphale de l'Epinoche (Cestodes). Mais cette remarquable découverte était tombée dans l'oubli. Il faudra attendre 1853 pour que P .J. Van Beneden établisse un rapport entre Taenia solium et Cysticercus cellulosae (Cestodes), 1873 (Weinland) et 1883 (R. Leuckart) pour que le cycle de Fasciola hepatica soit éclairci (Trématodes), 1882 (R. Leuckart) pour connaître le cycle de Strongyloïdes stercoralis (Nématodes), 1886 pour que Golgi saisisse le cycle schizogonique des Hématozoaires du paludisme (Sporozoaires) et 1899 - plus de 100 ans après Abildgaard - pour que Rabinowitsch et Kemper décrivent l'évolution du Trypanosoma lewisi (Flagellés). On notera que les Pentastomides sont assez bien placés dans l'évolution de nos connaissances, puisque le cycle de Linguatula serrata fut définitivement éclairci vers 1860 (R. Leuckart).

(2) En fait, H.-D. de Blainville reconnaît, parmi les Onchocéphalés, le seul genre « Linguatule » groupant « cinq genres qui n'en sont, évidemment, qu'un »: Linguatule, pentastome, tetragule, porocéphale, prionoderme. Il y ajoute le genre «cucullan» ( $\&$ trop mal connu pour assurer sa place »), le genre «nettorhynque » et le genre «caryophyllée» ( $\&$ trop mal connu pour juger de ses rapports naturels »).

Le genre «Nettorhynque » est un genre fabuleux que H.-D. de Blainville abandonnera dès 1828 . 
C'est à cette époque que P.-J. van Beneden commence ses remarquables travaux qui devaient amener, une quinzaine d'années plus tard, des conclusions nouvelles.

Car les descripteurs sont partagés entre deux tendances:

- grouper et définir les espèces, ce qui, en l'absence de toutes données sur les cycles parasitaires, entraîne des erreurs importantes (2 bis) ;

- classer le groupe parmi les ordres déjà définis.

A ce titre, les vieux auteurs sont unanimes: les Pentastomides sont des « vers » ou mieux des «Entozoaires »(3). Ils sont trompés par la convergence morphologique de ces parasites et des vers plats rubannés.

Pourtant, dès 1845, F. Dujardin émet des doutes sur cette assimilation :

« Les Acanthothèques présentent un certain rapport avec les crustacés entomos« tracés ou les crustacés parasites dont les appendices antérieurs seraient représentés « par les deux paires de crochets. Ils ont d'autres rapports avec les Nématoïdes et les « Trématodes, mais ils ne peuvent être convenablement rangés ni avec les uns, ni avec « les autres. »

Mais F. Dujardin ne reconnaît encore que le genre Pentastoma Rudolphi (= Linguatula Cuvier, Lamarck, Owen).

En 1849, P.-J. van Beneden découvre l'organisation très particulière de l'embryon et en souligne la nature arthropodiale. Les Linguatulides deviennent des Arthropodes de la classe des crustacés.

En 1852, Schubart propose de les placer dans les Acariens. Cette position est celle également de R. Leuckart, 1860 qui distingue la famille des Linguatulidae dans l'ordre des Acariens. Enfin, et le fait est capital, R. Leuckart élucide le cycle de Linguatula serrata.

Notons d'ailleurs que c'est souvent sur des homologies discutables que se fondent les opinions. On admet sans trop de peine que les crochets sont des pattes « atrophiées », on trouve une analogie «curieuse » entre les Pentastomides et les Acariens démodicides. Les ouvrages de vulgarisation (4) et des ouvrages ou articles plus spécialisés (5) reproduisent ces opinions.

( 2 bis) Ainsi, en 1840, M. Nordmann (Annotation à Lamarck), reprend le genre Linguatula de Lamarck sans apporter aucune lumière nouvelle. Il existe une véritable scolastique dans l'étude du groupe.

(3) Cette pensée sera encore en plein $\mathrm{xx}^{e}$ siècle celle de C.E.W. Sprehn (1932).

(4) Cf. entre autres Roule L., Suis A., Jammes L. 1895: « Cours de Zoologie générale et médicale », 2e éd., 369-370; Moniez R. 1896: "Traité de Parasitologie », 565 ; Neveu-Lemaire M. 1908: «Précis de Parasitologie humaine », $4^{e}$ édit., 522; Brumpt E. 1910: «Précis de Parasitologie », $1^{\text {re }}$ édit., 487; Neveu-Lemaire M. 1912: "Parasitologie des animaux domestiques », 862. Neveu-Lemaire M. 1921: "Précis de Parasitologie humaine », 5édit., 317; Brumpt E. 1927: «Précis de Parasitologie », 828; Franchin et Giordano 1929: «. Patologia tropicale »; NeveuLemaire M. 1938: «Traité d'Entomologie médicale et vétérinaire », 153-173; Brumpt E. 1949 : «Précis de Parasitologie », 6e édit., 1060-1065; Manson-Bahr H. 1950: « Manson's Tropical Diseases », 1013-1014.

(5) W. S. Patton et F.W. Gragg, 1913: «A text book of medical Entomology », Christian Litterature Society for India, London, Madras, Calcutta, admettent deux genres Porocephalus et

Ann. de Parasitologie humaine et comparée (Paris), t. 38, 1963, $\mathrm{n}^{\circ} 3$ 
Depuis 70 ans, quatre révisions générales méritent d'être relevées qui sont pour l'historien des Pentastomides autant d'étapes dans nos connaissances: A. E. Shipley, 1898, L. W. Sambon, 1922, R. Heymons, 1935, A. Fain, 1961.

La position systématique des Pentastomida a retenu l'attention bien entendu des biologistes. Il est évident que les Pentastomida ne sauraient être placés dans les Arachnides, au voisinage de groupes aussi différenciés que les Scorpionides, les Chernétides, les Solifuges, les Palpigrades, les Aranéides, les Acariens.

Dans ces conditions, L. Cuénot (1926, puis 1949 et 1952) les isola au voisinage des Tardigrades et des Onychophores dans les Malacopoda (= Pararthropoda) non sans de grosses hésitations. L. von Graff, sous le nom de Stelechopoda, unit les Linguatulides, les Myzostomes et les Tardigrades. Enfin, en 1949, A. Vandel souligne l'hétérogénéité certaine de ces groupes.

\section{Les genres et les espèces}

La taxinomie actuelle des Pentastomides est l'œuvre de L. W. Sambon et de R. Heymons.

Nous avons résumé, sous forme de tableaux synoptiques immédiatement abordables, l'essentiel de nos connaissances.

Il existe deux groupes très différents: les Cephalobaenida primitifs et les Porocephalida plus évolués (tableau 1).

- Les Cephalobaenida groupent deux familles Cephalobaenidae et Reighardiidae (tableau 2). Les Cephalobaenidae renferment deux genres certains et un genre douteux (tableau 3). L'un de ces genres, le genre Raillietiella, est complexe et deux classifications ont été proposées (tableau 4).

- Les Porocephalida se divisent en deux superfamilles: Porocephaloïdea et Linguatuloïdea (tableau 5).

Les Porocephaloïdea réunissent cinq familles différentes: Sebekiidae, Subtriquetridae, Samboniidae, Porocephalidae, Armilliferidae (tableau 6). L'analyse des principales familles est donnée dans les tableaux 7, 8, 9 et 10 .

Enfin, nous dresserons le catalogue des espèces actuellement reconnues (tableau 11).

Linguatula, placés à côté des Arachnides. L. W. Sambon, 1922 considère les Pentastomides comme des Acariens, F. Noc, 1923 également en s'appuyant sur des données évolutives (embryon acariforme). Les Pentastomides sont classés par cet auteur au voisinage des Demodécides et des Pycnogonides. V. Haffiner, 1924, après étude anatomique, les rapproche soit des Acariens, soit des Myriapodes, soit des Onychophores. 


\section{Tableau 1.}

\begin{tabular}{|c|c|c|}
\hline & CEPHALOBAENIDA & POROCEPHALIDA \\
\hline $\begin{array}{l}\text { Embryon: } \\
\text { « pattes } \gg \ldots \ldots \ldots \ldots \\
\text { appareil perforant } \ldots \ldots \ldots \\
\text { \& queue } » \ldots \ldots \ldots \ldots \ldots\end{array}$ & $\begin{array}{l}\text { segment basal en anneau. } \\
\text { stylet médian bifurqué, } 3 \text { pai- } \\
\text { res de petits aiguillons laté- } \\
\text { raux. } \\
\text { longue, bifide. }\end{array}$ & $\begin{array}{l}\text { un axe chitineux terminé en } \\
\text { cupule. } \\
\text { stylet médian simple, } 1 \text { paire } \\
\text { de stylets latéraux bifides } \\
\text { (en Y), avec glande annexe. } \\
\text { courte, à } 2 \text { pointes mousses. }\end{array}$ \\
\hline $\begin{array}{l}\text { Larve }(\lambda \text { III }): \\
\text { griffes } \ldots \ldots \ldots \ldots \ldots \\
\text { lobes parapodiaux } \ldots \ldots \ldots \\
\text { aiguillons céphaliques } \ldots \ldots \\
\text { épines abdominales } \ldots \ldots\end{array}$ & $\begin{array}{l}2 \text { griffes égales. } \\
\text { présents. } \\
\text { nombreux. } \\
\text { absentes. }\end{array}$ & $\begin{array}{l}\text { grife simple (ou double. mais } \\
\text { alors griffe accessoire dor- } \\
\text { sale). } \\
\text { absents. } \\
\text { absents. } \\
\text { une couronne de petites épines } \\
\text { (souvent mousses) au bord pos- } \\
\text { térieur des anneaux. }\end{array}$ \\
\hline $\begin{array}{l}\text { poche du cirre } \ldots \ldots \ldots \ldots \\
\text { cirre } \ldots \ldots \ldots \ldots \ldots \ldots \ldots \\
\text { gubernaculum } \ldots \ldots \ldots \ldots \\
\text { utérus } \ldots \ldots \ldots \ldots \ldots\end{array}$ & $\begin{array}{l}\text { non fusionné. } \\
\text { simples sans fulcrum. } \\
\text { primitive, en arrière de la } \\
\text { bouche. } \\
\text { présents ou absents. } \\
\text { antérieur, ventral. } \\
\text { antérieur, ventral (femelle pro- } \\
\text { gyne). } \\
\text { absente. } \\
\text { court, soudé au gubernaculum. } \\
\text { en tige chitineuse. } \\
\text { large, sacciforme. }\end{array}$ & $\begin{array}{l}\text { une masse indivise. } \\
\text { avec fulcrum. } \\
\text { en trapèze aplati, en ligne } \\
\text { courbe ou sur le même plan } \\
\text { que la bouche. } \\
\text { absents. } \\
\text { antérieur, ventral. } \\
\text { postérieur, soit sur l'anneau } \\
\text { distal (femelle opisthogyne), } \\
\text { soit sur les anneaux précé- } \\
\text { dents (femelle hétérogyne). } \\
\text { présente. } \\
\text { long et libre. } \\
\text { en gouttière. } \\
\text { étroit, allongé, sinueux. }\end{array}$ \\
\hline
\end{tabular}


Tableau 2. - CEPHALOBAENIDA

\begin{tabular}{|c|c|c|}
\hline & Cephalobaenidae & Reighardiidae \\
\hline $\begin{array}{l}\text { Lobes podiaux ou parapodiaux } \\
\text { Griffes } \ldots \ldots \ldots \ldots \ldots \ldots \\
\text { Papilles frontales } \ldots \ldots \ldots \ldots \\
\text { Papilles céphaliques dorsales. } \\
\text { Extrémité postérieure } \ldots \ldots \ldots \\
\text { Cuticule } \ldots \ldots \ldots \ldots \ldots \ldots \\
\text { Nombre de genres } \ldots \ldots \ldots \ldots \\
\text { Nombre d'espèces } \ldots \ldots \ldots \ldots \\
\text { Hôtes des adultes } \ldots \ldots \ldots \ldots \\
\text { Hótes des larves } \ldots \ldots \ldots \ldots\end{array}$ & $\begin{array}{l}\text { présents. } \\
\text { moyennes. } \\
\text { présentes. } \\
\text { présentes. } \\
\text { à deux lobes, anus terminal } \\
\text { entre les lobes. } \\
\text { lisse. } \\
\quad 33 \\
\quad 23 \\
\text { Serpents, Lacertiniens, ? Am- } \\
\text { phibiens. } \\
\text { Serpents, Amphibiens. }\end{array}$ & $\begin{array}{l}\text { absents. } \\
\text { très petites. } \\
\text { présentes, mais réduites. } \\
\text { absentes. } \\
\text { arrondie, non lobée. } \\
\text { petites élevures épineuses. } \\
\qquad 1 \\
1\end{array}$ \\
\hline
\end{tabular}

Tableau 3. - Cephalobaenidae

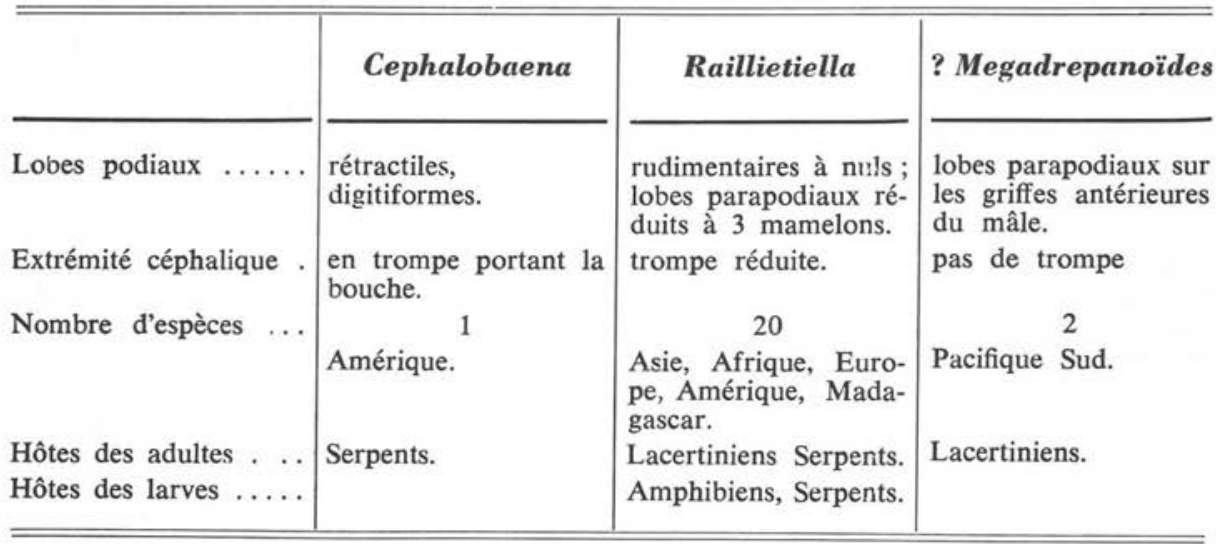

* La position du genre Megadrepanoïdes est incertaine : selon ses créateurs, ce genre réunirait des caractères de Cephalobaenida (femelle progyne) et des caractères de Porocephalida (utérus tubulaire, griffes présentant un fulcrum). 
Tableau 4. - Le genre Raillietiella.

\begin{tabular}{|c|c|c|c|c|c|}
\hline $\begin{array}{l}\text { Classification de } R \text {. Heymons } \\
\text { Groupes } \ldots \ldots \ldots \ldots \ldots \ldots\end{array}$ & I & II & III & IV & V \\
\hline $\begin{array}{l}\text { Classification de M.-L. Hett } \\
\text { Sous-genres } \ldots \ldots \ldots \ldots \ldots\end{array}$ & Heymonsia & \multicolumn{4}{|c|}{ Raillietiella } \\
\hline $\begin{array}{l}\text { Corps } \ldots \ldots \ldots \\
\text { Griffes antérieures. } \\
\text { Lobes podiaux } \ldots\end{array}$ & $\begin{array}{l}\text { en fuseau. } \\
\text { réduites. } \\
\text { rudimentaires }\end{array}$ & \multicolumn{4}{|c|}{$\begin{array}{l}\text { allongé. } \\
\text { normales. } \\
\text { nuls. }\end{array}$} \\
\hline Lobes postérieurs. & & $\begin{array}{l}\text { épais } \\
\text { charnus }\end{array}$ & $\begin{array}{l}\text { courts } \\
\text { larges }\end{array}$ & $\begin{array}{l}\text { petits } \\
\text { globuleux }\end{array}$ & allongés \\
\hline $\begin{array}{l}\text { Nombre d'espèces } \\
\text { Hôtes des adultes } \\
\text { Hôtes des larves. }\end{array}$ & $\begin{array}{c}\quad 6 \\
\text { Lézards } \\
\text { ? Amphibiens }\end{array}$ & $\begin{array}{c}2 \\
\text { Varans }\end{array}$ & $\begin{array}{c}\quad 4 \\
\text { Serpents } \\
\text { Serpents }\end{array}$ & $\begin{array}{c}\text { 2 } \\
\text { Serpents } \\
\text { Amphibiens }\end{array}$ & \begin{tabular}{l}
$\quad \quad 6$ \\
\multicolumn{1}{c}{$\quad$ Lacertiniens } \\
Serpents
\end{tabular} \\
\hline
\end{tabular}

* Le sous-genre Heymonsia ne correspond qu'imparfaitement au groupe I: R. gehyrae de Java en est exclu

Tableau 5. - PCROCEPH ALIDA

\begin{tabular}{|c|c|c|}
\hline & POROCEPHALOIDEA & LINGUATULOIDEA \\
\hline $\begin{array}{l}\text { Corps } \ldots \ldots \ldots \ldots \ldots \ldots \ldots \\
\text { Testicules } \ldots \ldots \ldots \ldots \ldots \ldots\end{array}$ & $\begin{array}{l}\text { cylindrique. } \\
\text { un seul. }\end{array}$ & $\begin{array}{l}\text { lancéolé, plat. } \\
\text { deux, } \\
4 \text { griffes simples, } \\
\text { bouche entre les griffes, } \\
\text { anus terminal, } \\
\text { vulve terminale, } \\
\text { nymphe à griffes doubles et } \\
\text { cuticule épineuse. }\end{array}$ \\
\hline $\begin{array}{l}\text { Hôtes des adultes } \ldots \ldots \ldots \ldots \\
\text { Hôtes des larves } \ldots \ldots \ldots \ldots \ldots\end{array}$ & $\begin{array}{l}\text { Reptiles. } \\
\text { Poissons, Reptiles, Mammifè- } \\
\text { res, rarement Oiseaux. } \\
5 \text { familles. }\end{array}$ & $\begin{array}{l}\text { Mammifères. } \\
\text { Mammifères. } \\
\text { une seule famille, } \\
\text { un genre, } \\
4 \text { espèces, }\end{array}$ \\
\hline
\end{tabular}




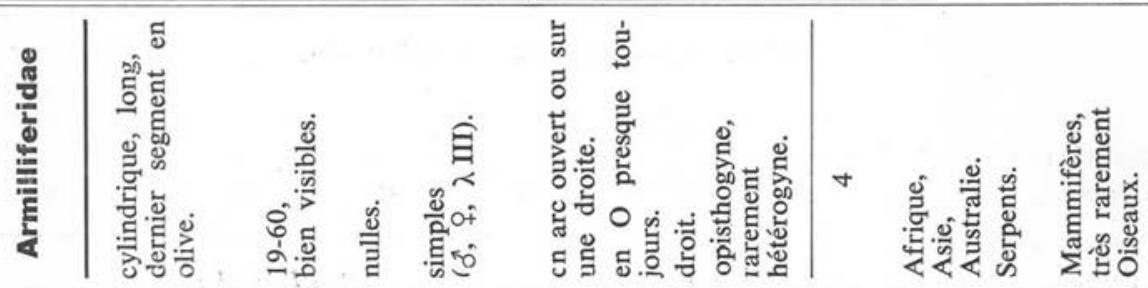

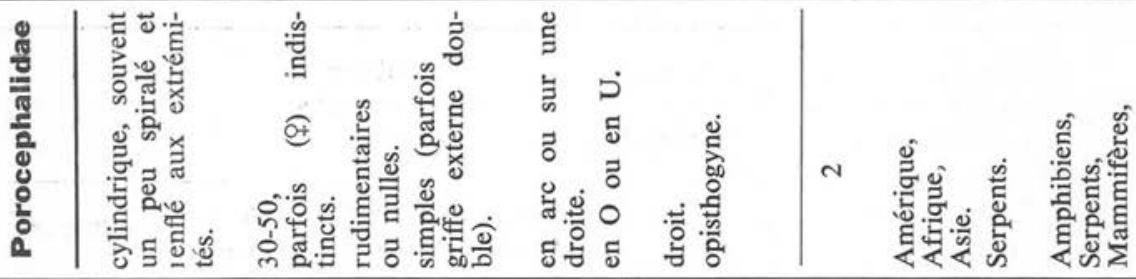


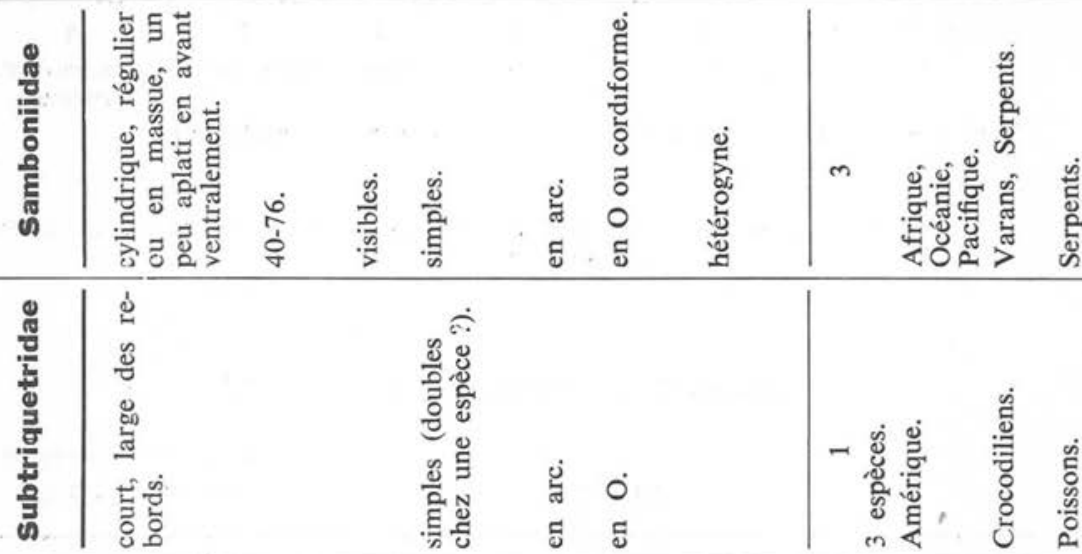

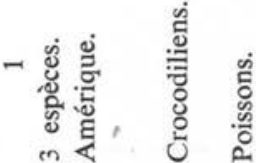

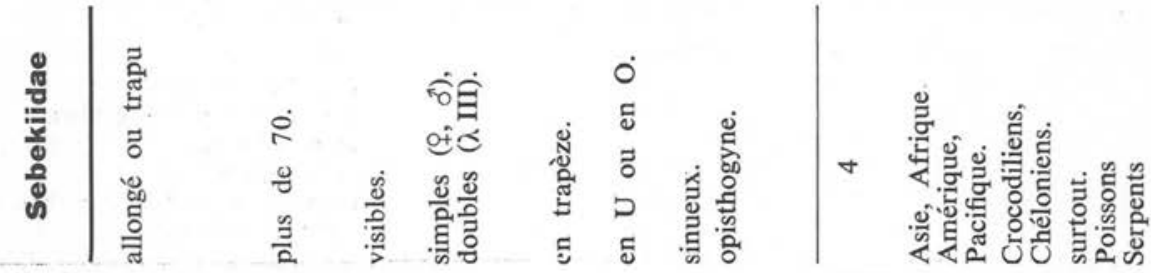

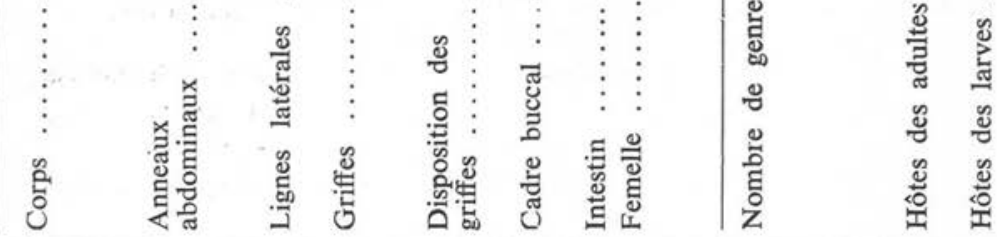




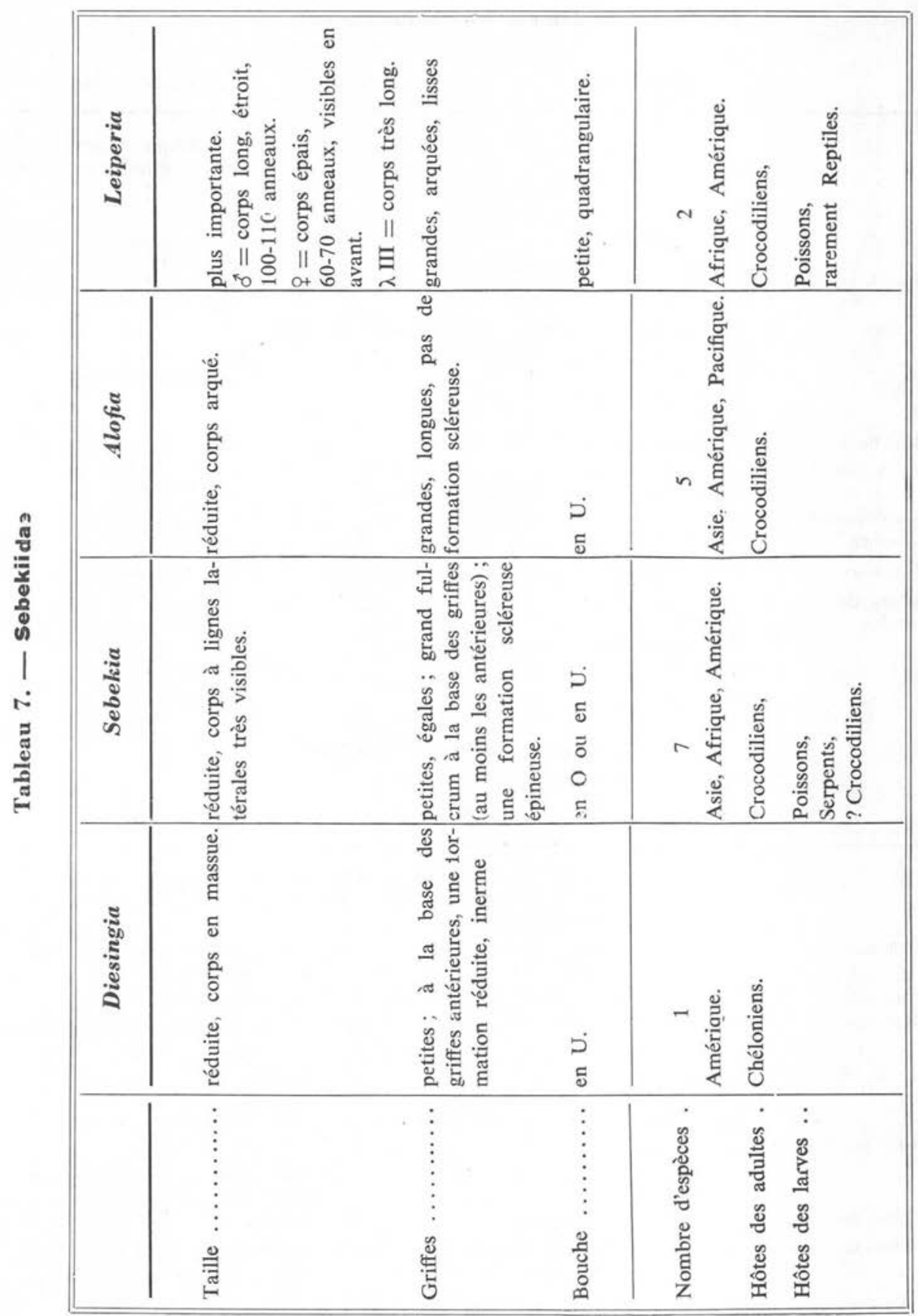


Tableau 8. - Samboniidae

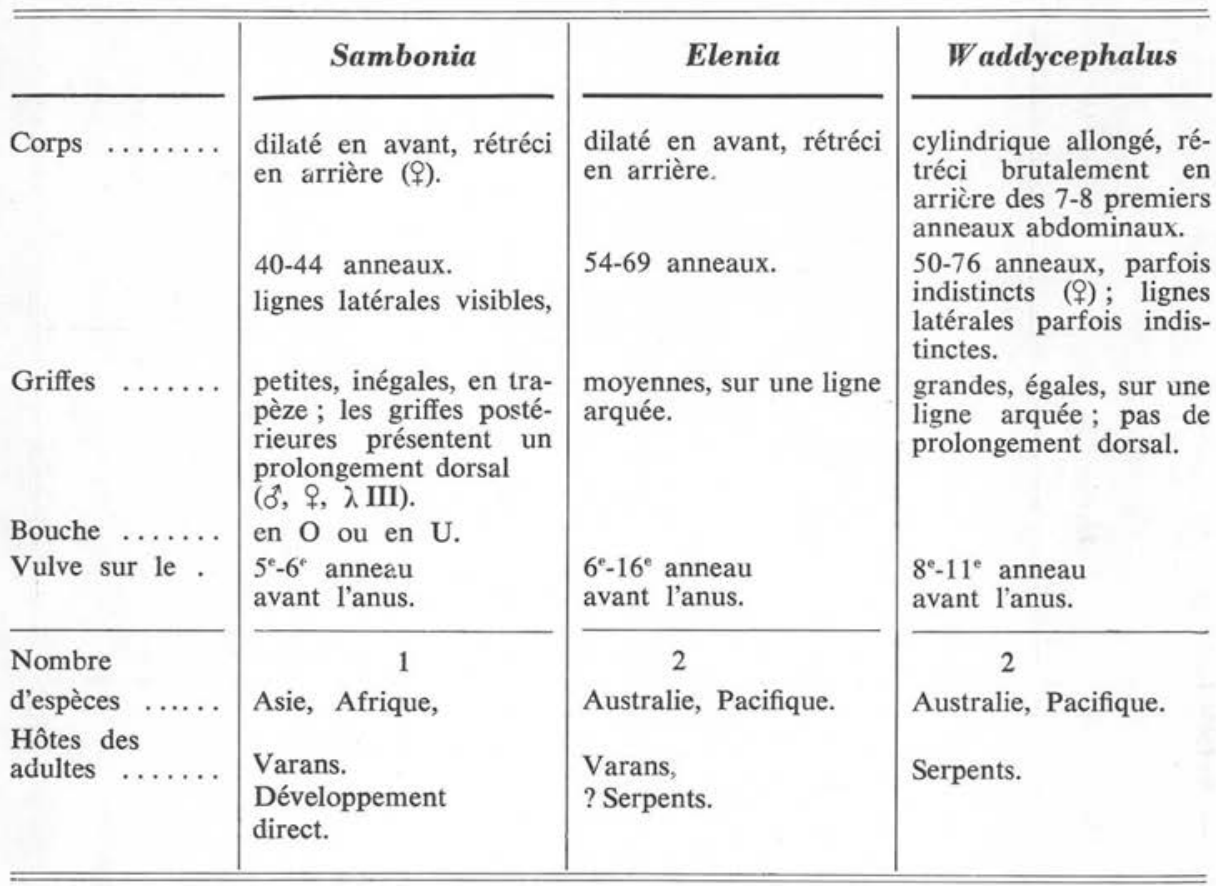

Tableau 9. - Porocephalidae

\begin{tabular}{|c|c|c|}
\hline & Porocephalus & Kiricephalus \\
\hline $\begin{array}{l}\text { Anneaux } \ldots \ldots \ldots \ldots \ldots \\
\text { Bouche } \ldots \ldots \ldots \ldots \ldots \ldots \\
\text { Griffe interne } \ldots \ldots \ldots \ldots \ldots \\
\text { Griffe externe } \ldots \ldots \ldots \ldots\end{array}$ & 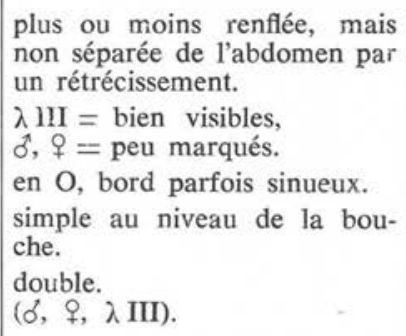 & $\begin{array}{l}\text { rerflée et séparée de l'abdo- } \\
\text { men par un rétrécissement. } \\
\text { corps cylindrique régulier, par- } \\
\text { fois spiralé ( }() \text { ). } \\
\text { en ovale allongé. } \\
\text { simple, un peu en avant de la } \\
\text { bouche. } \\
\text { simple. }\end{array}$ \\
\hline $\begin{array}{l}\text { Nombre d'espèces } \ldots \ldots \ldots \ldots \\
\text { Hôtes des adultes } \ldots \ldots \ldots \ldots \\
\text { Hôtes des larves } \ldots \ldots \ldots \ldots\end{array}$ & $\begin{array}{l}\quad 5 \\
\text { Afrique, Amérique. } \\
\text { Serpents. } \\
\text { Reptiles, Mammifères. }\end{array}$ & $\begin{array}{l}\quad 3 \\
\text { Asie, Amérique, Madagascar, } \\
\text { Australie. } \\
\text { Serpents. } \\
\text { Amphibiens, Reptiles, Mam- } \\
\text { mirères }\end{array}$ \\
\hline
\end{tabular}




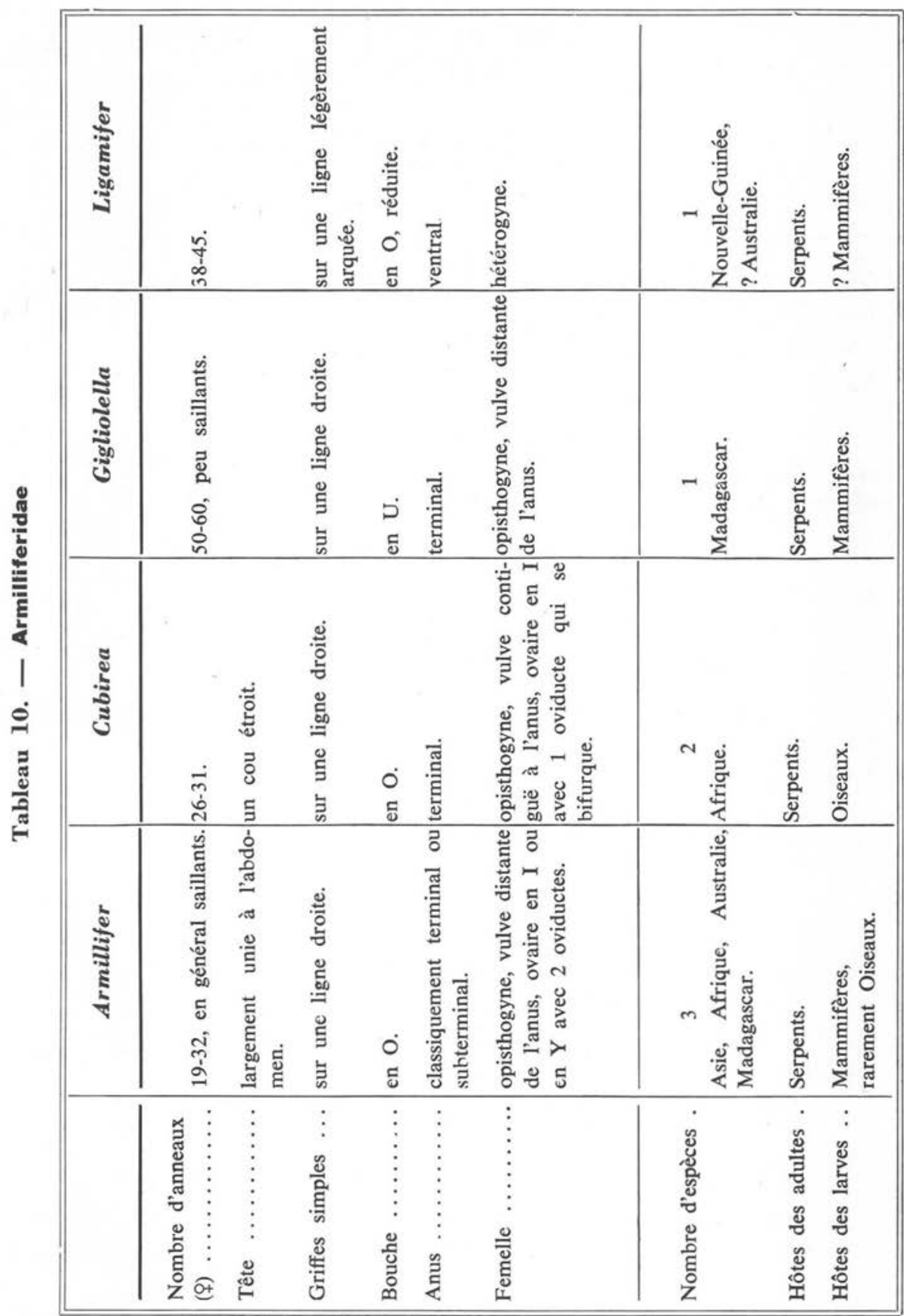




\section{Tableau 11. - Catalogue des espèces actuellement reconnues dans le phylum des PENTASTOMIDA}

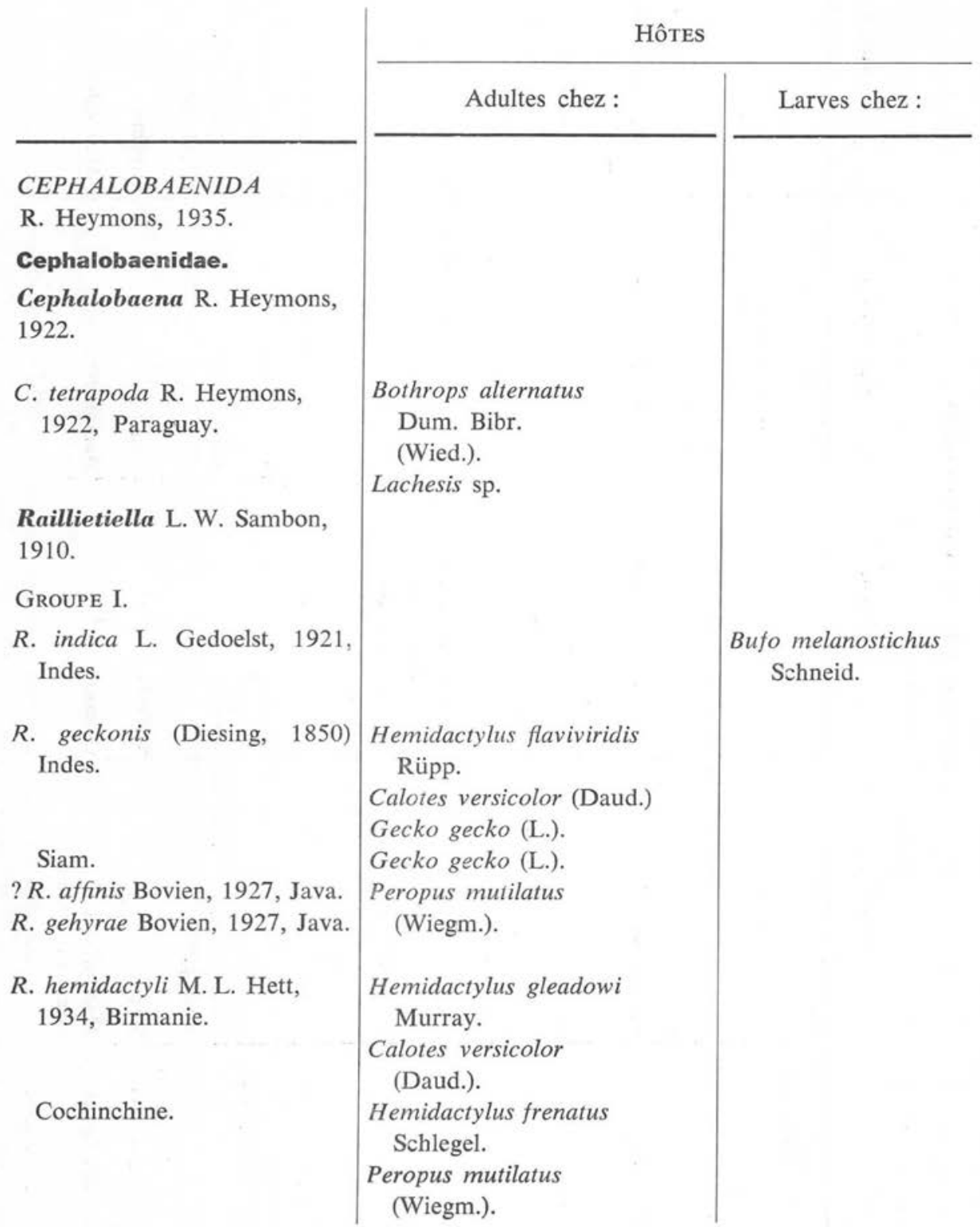


HôTES

\begin{tabular}{l|l}
\hline Adultes chez: & Larves chez :
\end{tabular}

R. mabuyae R. Heymons, 1922, Mabuya sulcata Peters. Afrique S.-O.

GROUPE II.

R. shipleyi R. Heymons、1926, Afrique.

Varanus exanthematicus Bosc.

Varanus griseus Daudin.

? Asie S.

Varanus ocellatus Rüpp.

R. kochi R. Heymons, 1926, Varanus ocellatus Rüpp. Abyssinie.

Groupe III.

$R$. boulengeri (Vaney et L. W. Sambon, 1910), Afrique.

R. boulengeri spiralis R. Hey-

Python regius (Shaw).

Bitis gabonica Dum.

Mehelya lamani

(Lönnh.).

Bitis nasicornis Shaw.

Bitis arietans Merr.

Causus rhombeatus

(Licht.).

Thrasops jacksoni Günth.

Thelotornis kirtlandi (Hall.).

Dromophis lineatus (Dum. Bibr.).

Psammophis sibilans

(L.).

Crotaphopeltis hotamboeia (Laur.).

Zamenis ravergieri Menet.

Psammophis sibilans L.

Psammophylax rhombeatus $\mathrm{L}$.

Psammophylax variabilis Gohr.

Causus rhombeatus (Licht.).

Boaedon olivaceus (Dum.).

Boaedon lineatus Dum. Bibr.

Naja melanoleuca Hall.

Naja nigricollis Reinh.

Sepedon haemachaetes Merr.

Dendroaspis jamesoni

(Traill.).

mons, 1939, Soudan.

Psammophis sibilans (L.).

Gastropyxis

smaragdina

(Schl.).

Mabuya. 


\begin{tabular}{|c|c|c|}
\hline & \multicolumn{2}{|c|}{ Hôtes } \\
\hline & Adultes chez: & Larves chez: \\
\hline $\begin{array}{l}\text { R. orientalis (M. L. Hett, 1915), } \\
\text { Asie E., Europe S. }\end{array}$ & $\begin{array}{l}\text { Coluber, Elaphe, Naja, } \\
\text { Ancistrodon, }\end{array}$ & ? Naja. \\
\hline $\begin{array}{l}\text { R. agcoi Tubangui et Masilun- } \\
\text { gan, 1936, Philippines. }\end{array}$ & Naja. & \\
\hline GROUPE IV. & . & \\
\hline $\begin{array}{l}\text { R. mediterranea (M. L. Hett. } \\
\text { 1915), Méditerranée. }\end{array}$ & $\begin{array}{l}\text { Zamenis dahli Fitz. } \\
\text { Zamenis gemonensis } \\
\text { (Laur.). }\end{array}$ & $\begin{array}{l}\text { Bufo mauretanicus } \\
\text { Schl. }\end{array}$ \\
\hline $\begin{array}{l}\text { R. spiralis M. L. Hett, } 1923 \text {, } \\
\text { Palestine. }\end{array}$ & $\begin{array}{l}\text { Caelopeltis monspessulana } \\
\text { Herm. }\end{array}$ & \\
\hline Groupe V. & & \\
\hline $\begin{array}{l}\text { R. congolensis A. Fain, } 1960, \\
\text { Congo ex-belge. }\end{array}$ & Thelotornis oatesi (Gtr.). & \\
\hline $\begin{array}{l}\text { R. furcocerca (Diesing, 1835), } \\
\text { Amérique S. }\end{array}$ & $\begin{array}{l}\text { Boa, Coluber, Elaphe, } \\
\text { Spilotes, Drymobius, } \\
\text { Lachesis, Phrynomax, } \\
\text { Rhadinacea. }\end{array}$ & \\
\hline $\begin{array}{l}\text { R. bicaudata R. Heymons et } \\
\text { Gr. Vitzthum, 1935, Améri- } \\
\text { que N. }\end{array}$ & $\begin{array}{l}\text { Elaphe carais coupeeri } \\
\text { Holbr. } \\
\text { Ophiobolus getula } \mathrm{L} .\end{array}$ & \\
\hline $\begin{array}{l}\text { R. schoutadeni A. Fain, 1960, } \\
\text { Congo ex-belge. }\end{array}$ & $\begin{array}{l}\text { Monopeltis schoutedeni } \\
\text { Witte. }\end{array}$ & \\
\hline $\begin{array}{l}\text { R. chamaeleonis Grétillat et } \\
\text { Brygoo, 1959, Madagascar. }\end{array}$ & $\begin{array}{l}\text { Chamaeleon oustaleti } \\
\text { Mocq. } \\
\text { Chamaeleon verrucosus } \\
\text { G. Cuv. }\end{array}$ & \\
\hline $\begin{array}{l}\text { R. giglioli M. L. Hett, 1924, } \\
\text { Amérique S. }\end{array}$ & Amphisbaena alba $\mathrm{L}$. & \\
\hline
\end{tabular}


? Megadrepanoüdes Self

et Kuntz, 1957.

M. varani Self et Kuntz, 1957, Pacifique $\mathrm{S}$.

M. salomonensis Self et Kuntz, 1957, Pacifique S.

\section{Reighardiidae.}

Reighardia Ward, 1899.

R. sternae (Diesing, 1864), Europe, Asie, Amérique.

POROCEPHALOIDEA

A. Fain, 1961.

POROCEPHALIDA

R. Heymons, 1935.

\section{Sebekiidae.}

Diesingia L. W. Sambon, 1922.

D. megastoma (Diesing, 1836), Brésil.

Sebekia L. W. Sambon, 1922.

S. jubini (Vaney et L. W. Sambon, 1910), Asie S.

S. wedli Giglioli, 1922, Afrique.

S. cesarisi Giglioli, 1922, Afrique.

S. oxycephala (Diesing, 1836), Amérique.

S. divestei Giglioli, 1922, Amérique.

S. samboni Travassos, 1924, Brésil.

S. acuminata Travassos, 1924, Brésil.

\section{HôTES}

Adultes chez:

Varanus indicus (Daud.).

Varanus indicus (Daud.).

Sternidae (Goélands et Sternes).

Hydraspis geoffroyana

(Schweigger).

(A été signalé à tort aux

Indes sur un Crocodilien).

Crocodilus siamensis Sch.

Crocodilus vulgatus

(G. Cuv.).

Crocodilus.

Crocodilus.

Crocodilus.

Crocodilus.

\section{Crocodilus.}

Les larves du genre Sebekia se développent chez les

Poissons d'eau douce.
Larves chez :

Poissons probablement.

(C. W. Stiles et A. Hassal, 1927). 


\section{HôTES}

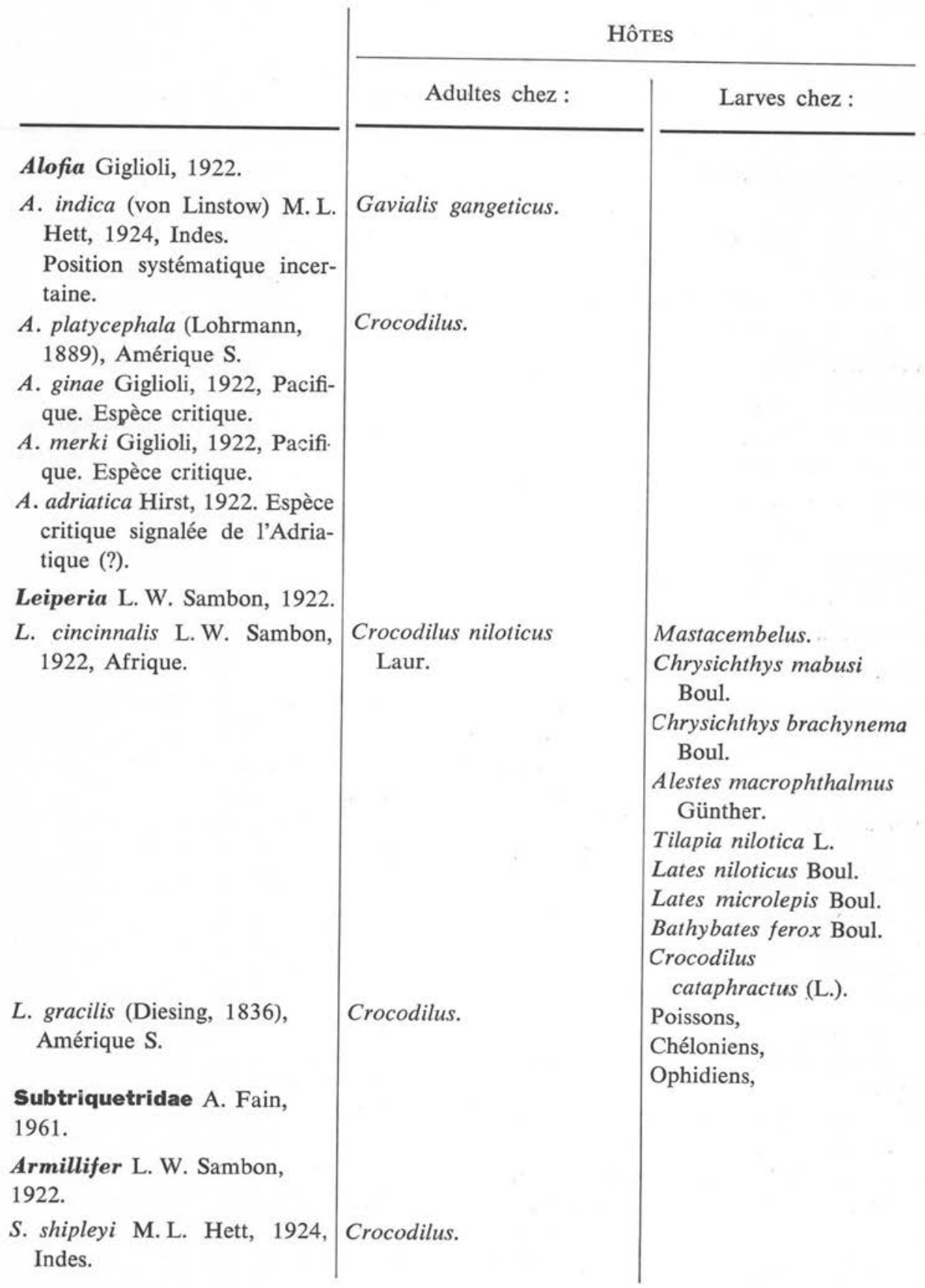


S. subtriquetra (Diesing, 1835), Brésil.

\section{Samboniidae.}

Sambonia F. Noc et Giglioli, 1922.

S. lohrmanni (L. W. Sambon, 1910), Asie, Afrique.

A été signalé par Self et Kuntz, 1957 d'un Ophidien des Iles Salomon (?).

Elenia R. Heymons, 1932.

E. australis R. Heymons, 1932, Australie.

E. travassosi (R. Heymons, 1932), Iles Philippines. Espèce critique.

Waddycephalus L. W.

Sambon, 1922.

W. teretiusculus (Baird, 1862), Australie.

W. vitiensis R. Heymons, 1932, Iles Fidji. Espèce critique.

\section{Porocephalidae.}

Porocephalus M. de

Humboldt, 1809.

P. subulifer (R. Leuckart, 1860), Afrique.

\section{HôTes}

Adultes chez:

Crocodilus crocodilus (L.).

Poissons d'eau douce.

Varanus.

Larves chez :

Varanus,

Pseudechis, Hoplocephalus, Notechis, Diemenia.

Mehelya poensis

A. Smith.

Mehelya savorgnani

(Mocq.).

Mehelya lamani (Lönnb.).

Développement interrompu probablement chez Bitis, Causus, Naja.
Développement direct possible.

Pseudechis.

Causus rhombeatus

(Licht.).

Psammophis sibilans (L.)

Neusterophis.

Elapsoïdea.

Cercopithecus.

Galago senegalensis

E. Geof. 


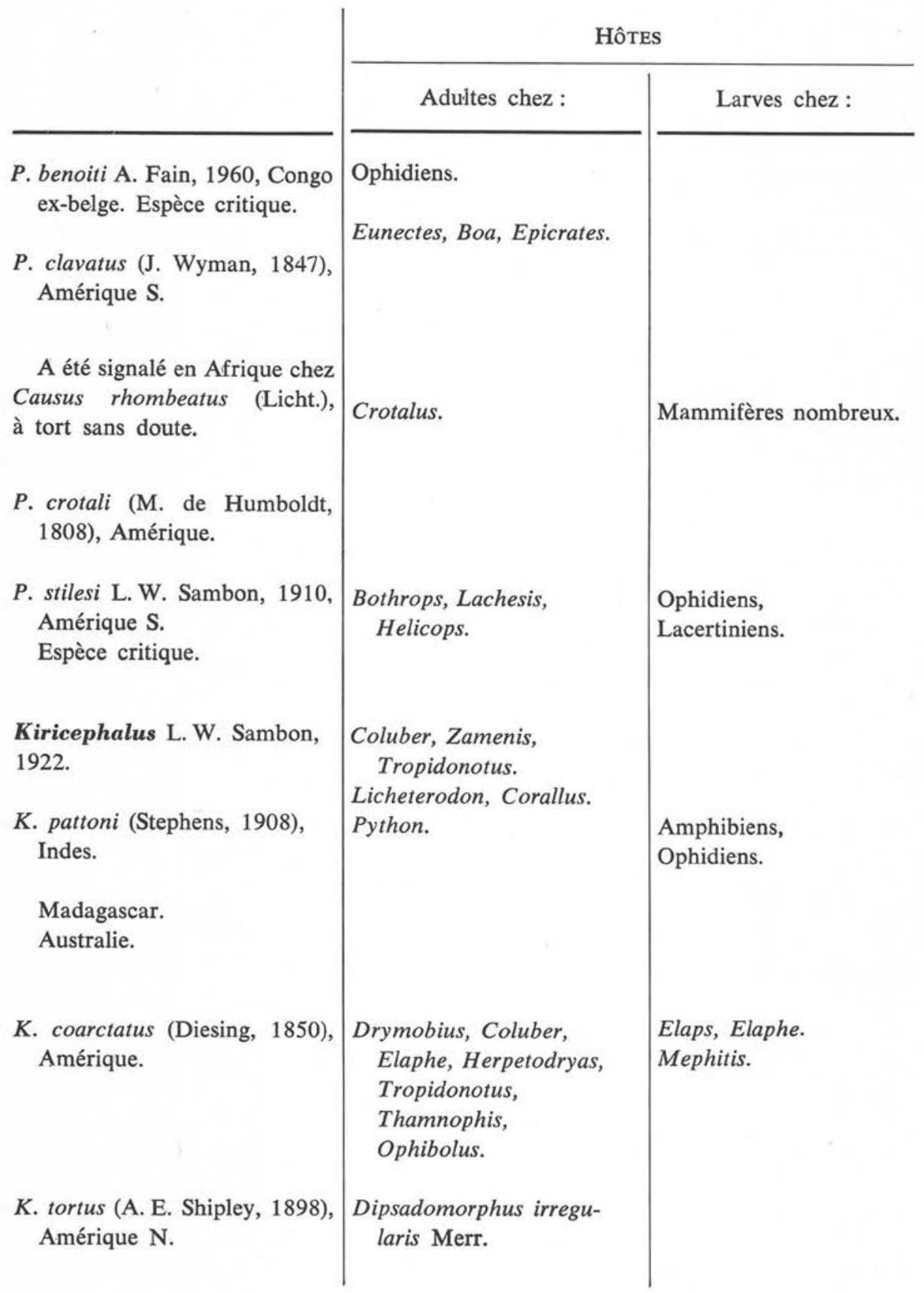




\section{HôTES}

Nettorhynchidae nom. nov. Nettorhynchus H.-D. de Blainville, 1824.

A. moniliformis (Diesing, 1835), espèce complexe:

A. m. moniliformis (Diesing, 1835),

Indes, Indomalaisie.

A été signalé en Afrique chez Python sebae (Gmel.).

A. m. heymonsi (L. W. Sambon, 1922), Indomalaisie, Indochine.

A. m. australis (R. Heymons, 1932), Australie.

A. armillatus (J. Wyman, 1847), Afrique.

Python reticulatus

Schneid.

Adultes chez :

Larves chez :

Python molurus L.

Mammifères nombreux.

Python amethystinus

Schneid.

Tropidonotus.

Python sebae (J.-F. Gmel.).

Mammifères nombreux.

Python regius (Shaw).

Rarement Oiseaux.

Boaedon lineatum

Dum. Bibr.

Bothrophtalmus lineatus

(Pet.).

Naja haje (L.).

Sepedon haemachata

(Lacép.).

Bitis arietans (Merr.).

Bitis gabonica

(Dum. Bibr.).

Bitis nasicornis (Shaw).

Cerastes cornutus (L.).

(Voir leur liste dans l'article

de Nicoli R.-M. et Golvan Y.,

A. grandis (M.-L. Hett, 1915), Afrique.

Bitis nasicornis (Shaw).
Bitis gabonica
(Dum. Bibr.).
Cerastes cornutus (L.).

Porphyrio. 


\begin{tabular}{|c|c|c|}
\hline & \multicolumn{2}{|c|}{ Hôtes } \\
\hline & Adultes chez: & Larves chez: \\
\hline $\begin{array}{l}\text { Cubirea Kishida, } 1929 . \\
\text { C. annulata (Baird, 1853), } \\
\text { Afrique. }\end{array}$ & $\begin{array}{l}\text { Naja haje L. } \\
\text { Naja melanoleuca Hall. } \\
\text { Naja nigricollis Reinh. }\end{array}$ & Porphyrio. \\
\hline $\begin{array}{l}\text { C. pomeroyi (Woodland, 1921), } \\
\text { Afrique. } \\
\text { A été signalé dans le Paci- } \\
\text { ique par Self et Kuntz, 1957, } \\
\text { sans doute à tort. }\end{array}$ & $\begin{array}{l}\text { Naja melanoleuca Hall. } \\
\text { Naja nigricollis } \text { Reinh. } \\
\text { Naja atriceps Laur. }\end{array}$ & \\
\hline $\begin{array}{l}\text { Gigliolella Chabaud et } \\
\text { Choquet, } 1954 . \\
\text { G. brumpti (Giglioli, 1922), } \\
\quad \text { Madagascar. }\end{array}$ & $\begin{array}{l}\text { Corallus madagascariensis } \\
\text { Dum. Bib. } \\
\text { Boa madagascariensis } \\
\text { Dum. Bib. }\end{array}$ & Lémuriens. \\
\hline $\begin{array}{l}\text { Ligamifer R. Heymons, } 1932 . \\
\text { L. mazzai (L. W. Sambon, } \\
\text { 1922), Asie orientale, Nou- } \\
\text { velle-Guinée?, Australie. }\end{array}$ & Ophidiens. & Dasyurus. \\
\hline $\begin{array}{l}\text { LINGUATULOIDEA A. Fain } \\
1961 .\end{array}$ & & \\
\hline $\begin{array}{l}\text { Linguatulidae. } \\
\text { Linguatula Frölich, } 1789 .\end{array}$ & & \\
\hline $\begin{array}{l}\text { L. serrata Frölich, 1789, cos- } \\
\text { mopolite. } \\
\text { L. dingophila Johns 1910, } \\
\text { Australie. }\end{array}$ & $\begin{array}{l}\text { Carnivores surtout. } \\
\text { Canis dingo } \mathrm{Bl} \text {. }\end{array}$ & $\begin{array}{l}\text { Mammifères, rare chez } \\
\text { Carnivores. }\end{array}$ \\
\hline $\begin{array}{l}\text { L. nuttali L. W. Sambon, } 1922 \text {, } \\
\text { Afrique orientale. }\end{array}$ & Felis leo L. & $\begin{array}{l}\text { Cephalophus } \\
\text { coeruleus } \text { Sm. } \\
\text { Tragelaphus scriptus. } \\
\text { (Pallas). }\end{array}$ \\
\hline $\begin{array}{l}\text { L. recurvata (Diesing, 1850), } \\
\text { Amérique S. }\end{array}$ & Felis onza L. & $\begin{array}{l}\text { Artiodactyles, } \\
\text { Primates. }\end{array}$ \\
\hline
\end{tabular}




\section{Essai phylogénétique}

Comme toujours, une classification valable, c'est-à-dire phylogénétique, doit tenir compte de multiples données:

1) des données d'ordre spatial ;

2) des données d'ordre biologique ;

3) des données d'ordre morphologique, celles-ci dans une certaine mesure discutables chez un groupe essentiellement parasite.

\section{1) Données spatiales.}

Elles sont soit stratigraphiques, soit géographiques.

a) Données stratigraphiques. - Nous n'en possédons aucune sur les Pentastomides. Mais, l'arbre généalogique des hôtes reptiliens doit retenir notre attention. Il s'agit là d'un problème parallèle sur lequel nous ne pourrons malheureusement longuement insister.

b) Données géographiques. - Sans nous y attarder, car nous avons devant nous des espèces parasites qui ont suivi leurs hôtes au cours de leurs déplacements, nous noterons les points suivants:

- le genre Cephalobaena, officiellement le plus primitif, est strictement américain ;

- le genre Raillietiella s'étend sur tout le secteur gondwanien (Amérique, Afrique, Madagascar, Indes). Il manque cependant à notre connaissance en Australie, mais présente deux rameaux, l'un méditerranéen, l'autre vers l'ExtrêmeOrient ;

- le genre Reighardia est cosmopolite, il est d'ailleurs le seul groupe inféodé aux Oiseaux ;

- le genre Megadrepanoïdes est pacifique ;

- tous les Porocephaloïdea sont gondwaniens, curieuse répartition déjà notée par L. Giglioli (1922), et résumée dans le tableau de la page 504 ;

- le genre Linguatula est cosmopolite.

\section{2) Données biologiques:}

Flles portent essentiellement sur les cycles parasitaires et nous devons avouer que nos connaissances sont à ce sujet bien réduites. Toutefois, en excluant le développement direct, nous obtenons le tableau à double entrée de la page 505 . 


\begin{tabular}{|c|c|c|c|c|c|c|}
\hline & $\begin{array}{l}\text { Améri- } \\
\text { ques }\end{array}$ & Afrique & $\begin{array}{c}\text { Madagas- } \\
\text { car }\end{array}$ & Indes & $\begin{array}{c}\text { Austra- } \\
\text { lie }\end{array}$ & $\begin{array}{l}\text { extension } \\
\text { pacifique }\end{array}$ \\
\hline 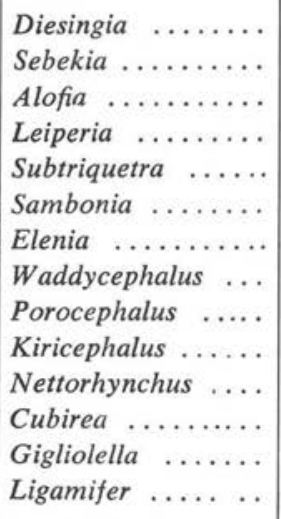 & $\begin{array}{l}+ \\
+ \\
+ \\
+ \\
+\end{array}$ & $\begin{array}{l}+ \\
+ \\
+\end{array}$ & $\begin{array}{l}+ \\
+\end{array}$ & $\begin{array}{l}+ \\
+ \\
+ \\
+ \\
\\
+ \\
+ \\
+ \\
+\end{array}$ & $\begin{array}{l}+ \\
+ \\
+ \\
+ \\
?+\end{array}$ & $\begin{array}{r}?+ \\
+\end{array}$ \\
\hline
\end{tabular}

Objectivement, nous devons reconnaître les faits suivants :

a) Le développement direct n'est actuellement connu sans équivoque que dans le genre Sambonia (A. Fain et J. Mortelmans, 1960) (6). Il est possible, mais non démontré, chez les Cephalobaenida parasites de petits lézards insectivores.

b) Les Pentastomides sont rarement des espèces sténoxènes. Cubirea pomeroyi semble strictement inféodé au genre Naja. Armillifer grandis serait capable de complet développement, uniquement chez Bitis nasicornis, mais il s'agit là peut-être d'une fausse impression due au petit nombre des serpents examinés. Porocephalus subulifer n'atteindrait également son complet développement que chez les Mehelya.

Ces espèces sont des espèces évoluées. Mais nous remarquerons que l'ubiquité de ces mêmes espèces à l'état larvaire est absolument extraordinaire. Nous connaissons ainsi plus de 60 espèces différentes capables d'héberger les larves d'Armillifer armillatus.

\section{3) Données morphologiques.}

Quelques questions doivent être soulevées:

\section{$1^{\circ}$ Nature de la mÉtamérisation.}

La métamérisation des Pentastomides est une fausse métamérisation, ce qui est d'ailleurs une donnée classique :

(6) Chez Sambonia lohrmanni Noc et Giglioli, parasite du Varanus komodoensis des Iles de la Sonde. L'exemplaire disséqué par Fain et Mortelmans leur a montré à la fois des formes adultes libres dans les cavités bronchiques et tous les stades de l'embryon à la nymphe enkystés dans la muqueuse trachéale. De nombreux œufs se trouvaient dans le mucus trachéal. 
$1=$ Pisces, 2 = Amphibia, $3=$ Reptilia, $31=$ Testudinata, $32=$ Loricata,

$33=$ Rhynchocephalia, $34=$ Sauria, $35=$ Ophidia, $4=$ Aves, $5=$ Mammalia

HôTES DÉFINITIFS

\begin{tabular}{|c|c|c|c|c|c|c|c|c|c|c|}
\hline & & 1 & 2 & 31 & 32 & 33 & 34 & 35 & 4 & 5 \\
\hline & 1 & & & & $\begin{array}{l}\text { Sebekia } \\
\text { Alofia } \\
\text { Leiperia } \\
\text { Subtriquetra }\end{array}$ & & & & Reighardia & \\
\hline & 2 & & & & & & $\begin{array}{l}\text { Railliet- } \\
\text { iella I? }\end{array}$ & $\begin{array}{l}\text { Railliet- } \\
\text { iella IV } \\
\text { Kiricephalus }\end{array}$ & & \\
\hline & 31 & & & & Leiperia & & & & & \\
\hline$\stackrel{g}{\mathscr{Q}}$ & 32 & & & & Leiperia & & & & & \\
\hline 㐭 & 33 & & & & & & & & & \\
\hline$\underline{z}$ & 34 & & & & & & & & & \\
\hline 总 & 35 & & & & Leiperia & & & $\begin{array}{l}\text { Railliet- } \\
\text { iella III } \\
\text { Waddycepha- } \\
\text { lus } \\
\text { Porocephalus } \\
\text { Kiricephalus }\end{array}$ & & \\
\hline & 4 & & & & & & & $\begin{array}{l}\text { Armillifer } \\
\text { Cubirea }\end{array}$ & & \\
\hline & 5 & & & & & & & $\begin{array}{l}\text { Porocephalus } \\
\text { Armillifer } \\
\text { Gigliolella } \\
\text { Ligamifer }\end{array}$ & & $\begin{array}{l}\text { Lingua- } \\
\text { tula }\end{array}$ \\
\hline
\end{tabular}

* Les hôtes intermédiaires sont inconnus chez les Diesingia (adultes chez les Testudinata), les Raillietella II et V, Megadrepanoïdes, Sambonia, Elenia (adultes chęz les Sauria), les Cephalobaena et Raillietella $V$ (adultes chez les Ophidia). 
a) la partie antérieure du corps est primitive: l'ancêtre métamérisé devait avoir une tête suivie de quatre ou cinq anneaux (L. Cuénot);

b) la partie postérieure du corps faussement métamérisée est due à l'énorme développement des organes génitaux, lui-même conséquence du parasitisme.

La preuve de cette fausse métamérie se trouve dans l'étude du système nerveux des formes primitives:

Chez les Cephalobaenida, la masse nerveuse céphalique est constituée par deux ganglions cérébroïdes unis par une commissure sous-œsophagienne. Le premier ganglion ventral est sous-œsophagien. Il existe au-delà trois ou quatre ganglions mal individualisés (R. Heymons).

Chez les Porocephalida, la condensation est totale: il n'existe plus qu'une seule masse ganglionnaire sous-œsophagienne, du moins chez l'adulte, car la larve présente encore des ganglions séparés.

Il faut souligner combien cette structure est évoluée. Les Onychophores présentent au contraire un système nerveaux beaucoup plus primitif, où l'on reconnaît facilement l'existence d'un complexe cérébroïde suivi d'une double chaîne de ganglions (une paire de ganglions par segment, ceux-ci étant indiqués par la position des lobopodes).

Les Tardigrades sont également primitifs : le complexe cérébroïde est, chez eux, suivi de quatre ganglions bilobés.

\section{$2^{\circ}$ La musculature.}

Les Pentastomides présentent une musculature striée développée. C'est là une acquisition importante que ne présentent ni les Onychophores, ni les Tardigrades, uniquement pourvus de muscles lisses.

\section{$3^{\circ}$ Signification Des crochets.}

Claus considérait les crochets comme les vestiges des deux dernières paires de pattes des Arachnides, W. Stiles comme les homologues des pièces buccales, F. Noc comme issus de l'évolution des deux paires de pattes de l'embryon.

Il s'agit là, en fait, d'une querelle sans objet. Les pièces buccales des Arthropodes, les pattes également sont issues, nous le savons aujourd'hui, d'une même origine. C'est ainsi que le groupe des Trilobitomorphes nous donne l'image ancestrale de ces formes déjà évoluées: autour de la bouche, les appendices paires du céphalon, biramés, évolueront vers les pièces buccales (Ch. Walcott, C.-E. Beecher). Il est, par conséquent, bien hasardeux d'établir des homologies en s'adressant à des organes très différemment évolués :

Les griffes des Pentastomides ne sont, parmi les groupes connus, comparables qu'aux griffes des lobopodes des Onychophores et aux diplogriffes des «pieds 》 des Tardigrades. Cette ressemblance est soulignée par l'existence des glandes annexes, qui ne sont pas sans analogie avec la glande crurale des Onychophores.

$4^{\circ}$ Organes sensoriels.

Le parasitisme a entraîné l'involution globale des organes sensoriels. Les yeux 
développés chez les Onychophores sont réduits, quand ils existent, à deux taches oculaires chez les Tardigrades. Ils manquent chez les Pentastomides.

\section{$5^{\circ}$ Appareil digestif.}

Il est constitué par un tube de triple origine: stomodaeum ectodermique avec anneau buccal, mesenteron endodermique et proctodaeum ectodermique. Une distribution analogue se retrouve chez les autres Arthropodes.

Il n'existe chez les Pentastomides aucune pièce buccale différenciée. Nous noterons toutefois que les Onychophores présentent une bouche à deux paires de lames mandibulaires qui sont l'analogue des griffes d'un membre disparu.

\section{$6^{\circ}$ Appareil circulatoire.}

Totalement involué chez les Pentastomides et les Tardigrades, l'appareil circulatoire existe chez les Onychophores sous la forme d'un cœur dorsal pourvu d'ostioles.

\section{$7^{\circ}$ Appareil respiratoire.}

Absent chez les Pentastomides et les Tardigrades, l'appareil respiratoire existe développé chez les Onychophores qui présentent des trachées et des vésicules coxales évaginables jouant un rôle branchial.

\section{$8^{\circ}$ APPAREIL EXCRÉteur.}

L'appareil excréteur est encore de type annélidien chez les Onychophores: à la base du lobopode s'ouvre un canal très court, issu d'une vésicule contractile, celle-ci recevant les sécrétions d'un tube contourné, le labyrinthe, prolongé d'un entonnoir à cils vibratiles, lui-même drainant une cavité terminale, le saccule.

Il existe également, dans la cavité générale, des athrocytes (grosses cellules ovoïdes à 1-3 noyaux en îlots) et des cellules péricardiales.

L'appareil excréteur est très involué chez les Tardigrades: glandes rectales de Malpighi (Vasa malpighii).

Chez les Pentastomides, nous ne relevons que les cellules pariétales, homologues probablement des athrocytes des Onychophores.

\section{$9^{\circ}$ Appareil génital.}

On trouve deux testicules chez les mâles et deux ovaires chez les femelles des Onychophores. L'ontogénèse de ces êtres est variable: les formes primitives présentent des œufs à vitellus abondant, mais il existe des espèces à œufs déméroblastisés dont le vitellus est très réduit.

On relève un seul testicule chez les mâles et un seul ovaire chez les femelles de Tardigrades. Ici, l'œuf holoblastique présente une segmentation totale et égale.

Cette segmentation totale se retrouve chez les Pentastomides dont l'œuf est vralsemblablement déméroblastisé. L'appareil génital lui-même est assez variable: ainsi, parmi les Porocephalida, oppose-t-on les Porocephaloïdea à testicule unique aux Linguatuloïdea à testicules séparés. 
Il y a là un point qui mérite notre attention: la segmentation spirale des Annélides se retrouve en partie dans la segmentation totale des Arthropodes primitifs (Xiphosures, Pantopodes, Entomostracés, Euphausiacés). Or, les Onychophores (en partie du moins), les Tardigrades et les Pentastomides présentent également cette segmentation totale. Mais est-elle réellement primitive pour ces trois groupes pararthropodiens ? Ceci est très discutable. Certains Onychophores, et ils appartiennent aux deux familles différentes: Peripatidae (genre Eoperipatus Evans) et Peripatopsidae (genre Peripatö̈des Pocok), ont un œuf centrolécithe qui subira une segmentation superficielle.

Les Tardigrades s'éloignent d'autre part de tous les Spiralia du fait de la formation du mésoderme par entérocœlie.

Tout se passe comme si nous étions en présence de plusieurs phylums différents et sans parenté: Onychophores, Tardigrades, Pentastomides, Arthropodes (ces derniers probablement polyphylétiques), tous ces phylums ayant très vraisemblablement cependant une origine commune lointaine responsable de multiples convergences.

En résumé, le tableau suivant, peut-être assez artificiel et incomplet, souligne la survivance des caractères annélidiens chez les trois groupes pararthropodiens:

\begin{tabular}{|c|c|c|c|}
\hline & ONYCHOPHORES & TARDIGRADES & Pentastomides \\
\hline \multicolumn{4}{|l|}{ C'aractères annélidiens: } \\
\hline $\begin{array}{l}\text { musculature lisse } \ldots . . . \ldots \\
\text { néphridies à entonnoir à }\end{array}$ & + & + & $\mathrm{O}$ \\
\hline cils vibratiles ............ & + & $\mathrm{O}$ & $\mathrm{O}$ \\
\hline $\begin{array}{l}\text { yeux formés d'une vésicule } \\
\text { close d'origine ectodermi- } \\
\text { que } \ldots \ldots \ldots \ldots \ldots\end{array}$ & + & O & $\mathrm{O}$ \\
\hline métamérie $\ldots \ldots \ldots \ldots \ldots$ & primitive. & $\begin{array}{l}\text { primitive, avec ré- } \\
\text { duction. }\end{array}$ & pseudométamérie. \\
\hline parapodes...$\ldots \ldots \ldots$. & lobopodes. & lobopodes. & $\begin{array}{l}\text { involution progressi- } \\
\text { ve des lobopodes. }\end{array}$ \\
\hline \multicolumn{4}{|l|}{ Caractères arthropodicns: } \\
\hline mues $\quad \ldots \ldots \ldots \ldots \ldots \ldots$ & $\stackrel{+}{+}$ & + & + \\
\hline appareil buccal..$\ldots \ldots$. & $\begin{array}{l}\text { différencié, } 2 \text { paires } \\
\text { de mandibules. }\end{array}$ & $\begin{array}{l}\text { en ventouse, avec } \\
\text { anneaux cuticulaires } \\
\text { lamellaires. }\end{array}$ & anneau scléreux. \\
\hline cœur à ostioles $\ldots \ldots \ldots$ & $\begin{array}{l}\stackrel{+}{\quad} \\
\text { cellules péricardiales } \\
\text { athrocytes. }\end{array}$ & $\mathrm{O}$ & $\begin{array}{c}\mathrm{O} \\
\text { cellules pariétales. }\end{array}$ \\
\hline cavité générale $\ldots \ldots \ldots$. & $\begin{array}{l}\text { réduite (saccules né- } \\
\text { phridiens, cavité go- } \\
\text { nadique). }\end{array}$ & $\begin{array}{l}\text { très réduite (cavité } \\
\text { gonadique). }\end{array}$ & persistante. \\
\hline système nerveux..$\ldots \ldots$ & primitif. & $\begin{array}{r}\text { réduction avec co } \\
\text { des }\end{array}$ & $\begin{array}{l}\text { escence progressive } \\
\text { nglions }\end{array}$ \\
\hline
\end{tabular}




\section{SYNTHESE}

Il nous faut maintenant établir une synthèse valable.

\section{$1^{\circ}$ Amcienneté des Pentastomides.}

En l'absence de tout document paléontologique, cette connaissance devrait nous être interdite. Or, les faits suivants nous permettent d'y suppléer dans une large mesure :

a) Les formes pararthropodiennes sont connues dès l'algonkien (précambrien). Xenusion auerswaldae Pompeckj, 1927, d'une quartzite de Scandinavie, était très probablement un Onychophore, ou peut-être le représentant d'un ordre méconnu et parallèle.

A cette époque existaient déjà, notons-le, des représentants des Annélides, des Crustacés et des Echinodermes.

Il s'ensuit nécessairement que les Pentastomides sont les contemporains de ces êtres et que leur ancienneté ne saurait être postérieure à l'algonkien.

b) Or, à cette époque, il n'existe aucun Vertébré, ceux-ci apparaîtront beaucoup plus tard, au silurien, en particulier au gothlandien supérieur (ludlovien). Ce sont les Ostracodermes, probablement voisins des Cyclostomes.

Dans ces conditions, force nous est ou d'admettre l'existence d'un parasitisme chez les Invertébrés, ou l'existence de formes libres. Nous connaissons certes un Tardigrade parasite (Tetrakentron synaptae L. Cuénot, 1892, qui perfore les tentacules du Leptosynapta galliennei Hérapath), mais il ne semble pas que ce soit là un fait déterminant, car il s'agit vraisemblablement d'une adaptation tardive.

Il y eut sans doute des formes libres, marines très probablement, qui n'ont pu survivre que par adaptation à la vie parasitaire. Cette adaptation est certainement tardive. A ces formes libres hypothétiques, très primitives, nous donnons provisoirement le nom de Palaeopentastomida. Nous pouvons, dans une certaine mesure, en tracer le schéma suivant: espèces (vraisemblablement de petite taille) métamérisées avec système nerveux primitif, mais en voie d'involution (coalescence des ganglions), lobopodes encore nombreux, existence d'un cœlome, de néphridies à cils vibratiles, segmentation spiralée. Ce tableau évoque, bien entendu, un Annélide. Mais, il y a là peut-être deux lignées parallèles. En fait, nous ignorons trop de choses : qu'était par exemple l'Helminthoidichnites, connu par des traces précambriennes? Tout ce que nous savons, c'est que, par la suite au silurien, existeront des Annélides évolués: Eunicites, Arabellites, Serpula et Spirorbis, et qu'à cet instant, les Palaeopentastomida devaient exister sous une forme libre, puisque les hôtes vertébrés n'étaient point encore apparus.

\section{$2^{\circ}$ Le parasitisme des Pentastomida.}

Nous avons vu que le cycle des Pentastomida est presque toujours un cycle dihétéroxène. Or, des remarques très particulières doivent être faites (dans la mesure, bien entendu, de nos connaissances encore réduites) :

1) Les Cephalobaenida sont des parasites des Ophidiens et des Lacertiniens, groupes essentiellement voisins. Mais, dans le genre Raillietiella, existent des formes qui parasitent à l'état larvaire les Amphibiens. D'autre part, la famille des Reighardiidae est 
parasite des Oiseaux de mer, à l'état adulte du moins, des Poissons, peut-être. à l'état larvaire.

En d'autres termes, ces formes semblent adaptées primitivement respectivement aux Amphibiens et aux Poissons et secondairement aux Reptiles et aux Oiseaux.

2) Or, si cette progression se retrouve chez certains Porocephalida (les Sebekia parasites à l'état larvaire des Poissons d'eau douce et à l'état adulte des Crocodiliens), la plupart des Porocephaloïdea sont inféodés aux Reptiles, le développement larvaire se faisant chez des êtres beaucoup plus évolués que les Reptiles (Mammifères).

3) Enfin, les Linguatulö̈dea sont essentiellement parasites des Mammifères.

Tout se passe comme s'il y avait eu des formes primitives inféodées aux Poissons et aux Amphibiens, qui par la suite ont atteint les Reptiles.

Ainsi, pouvons-nous dresser le tableau suivant:

\begin{tabular}{|c|c|c|c|}
\hline Poissons & Amphibiens & $\begin{array}{l}\text { Reptiles } \\
\text { Oiseaux }\end{array}$ & Mammifères \\
\hline
\end{tabular}

CEPHALOBAENIDA

formes larvaires

adultes

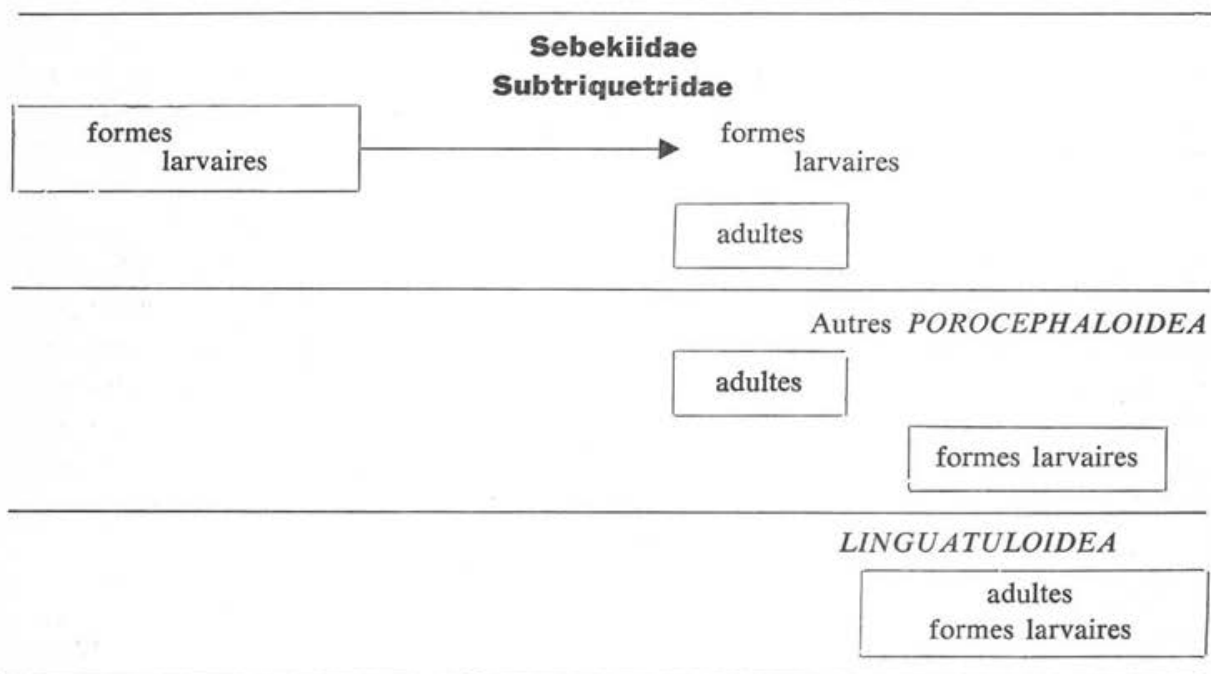

Il y a là plus qu'une simple coïncidence: les Reptiles constituent l'axe central actuel du groupe, mais leurs parasites sont venus des Poissons et des Amphibiens.

Il nous faut donc évoquer brièvement le destin des Vertébrés. 
Si les Cyclostomes sont inconnus à l'état fossile, les Ostracodermes en sont voisins et ils ont vécu entre le silurien supérieur et le dévonien, concurremment avec les Placodermes qui, nous le savons, sont souche des Elasmobranches. Crossopterygiens et Dipneustes (Choanates) apparaissent au dévonien et survivent encore.

Les Actinoptérygiens (Poissons supérieurs) sont connus (Chondrostéens) dès le permien, mais les Téléostéens sont plus tardifs (lias). Les Choanates sont à la base des Vertébrés tétrapodes. Ainsi, les Ichthyostegidae du dévonien supérieur, classés parmi les Stégocéphales, sont issus des Crossoptérygiens. Ce groupe des Stégocéphales précédera, du dévonien au trias, l'apparition des Urodèles (Crétacé supérieur) et des Anoures (Kimméridgien).

Nous reprendrons ici l'hypothèse de Säve-Söderbergh, 1934 : la classe des Choanates montre deux phylums distincts: $a$ ) le phylum des Dipneustes, qui donnera naissance aux Urodèles; $b$ ) le phylum des Crossoptérygiens qui, par l'intermédiaire des Stégocéphales et des Anoures, donnera naissance aux Amniotes.

La généalogie de ceux-ci mérite une brève évocation. Dès le carbonifère supérieur, trois phylums sont déjà individualisés :

— les Mésosauriens, source peut-être des Ichthyosauriens (trias, crétacé);

- les Pélycosauriens, dont la destinée sera brève, et qui s'éteindront au permien ;

- les Cotylosauriens enfin dont l'avenir sera tout différent et qui donneront naissance à de nombreux rameaux :

- Sauroptérygiens (trias-crétacé inférieur);

- Chéloniens (permien-actuel) ;

- Eosuchiens (permien-jurassique);

- Ornithosuchiens (trias), source des Lacertiniens (trias-actuel), et des Ophidiens (trias-actuel), des Rhynchocéphaliens (trias-actuel), des Sauropodiens (trias-crétacé), des Ornithisciens (jurassique-crétacé), des Crocodiliens (jurassique-actuel), des Ptérosauriens (jurassique-crétacé), des Oiseaux enfin (jurassique-actuel);

- Thérapsidiens (permien-trias), souche des Mammifères (trias-actuel).

La filiation des Pentastomides nous apparaît, semble-t-il, maintenant plus clairement :

Un premier rameau a dû s'adapter aux Actinoptérygiens et aux Crossoptérygiens (au dévonien-permien), puis s'épanouir parmi les Stégocéphales pour gagner par la voie des Ornithosuchiens les Lacertiniens et les Ophidiens. Les Cephalobaenida actuels en sont les lointains survivants.

Mais, un second rameau (parallèle, ou issu du précédent, nous l'ignorons absolument) a surgi parmi les Cotylosauriens beaucoup plus tard (au permien-trias). Son destin devait être infiniment plus varié : les Porocephalida actuels si hétérogènes évoquent une histoire mouvementée.

Le groupe a évolué durant la fragmentation de la Gondwanie. Nous commençons à peine à saisir les différentes phases de cette évolution. 
Le règne des Cestodes devait, par une sorte de balancement, amener l'extinction des Pentastomides. Les deux groupes parasites ont entre eux une homologie qui dépasse largement le cadre de la zoologie classique.

D'un groupe qui a été sans aucun doute puissant et varié au secondaire, nous ne connaissons aujourd'hui que quelques espèces relictes dont la valeur pour nous est considérable: ce sont les témoins d'un monde disparu.

\section{Bibliographie}

AbIldgaARD, 1789. - Zool. dan., III, 52 pl., 108.

Blainville (H.-D. DE), 1824. - Appendice au « Traité zoologique et physiologique sur les vers intestinaux de l'homme », par M. Bremser; trad. Grundler, Paris, 501-553, Atlas.

Blanchard (R.), 1888-1890. - Traité de Zoologie médicale, II.

Bosc, 1811. - Bull. Soc. Philomatique, $\mathrm{n}^{\circ} 44,269$, pl. 2, fig. 1.

Breinl (A.) et Hindle (E.), 1909. - A new Porocephalus (P. cercopitheci n. sp.). Ann. Trop. Med. and Paras., II.

BRUES (C. T.), 1942. - Occurence of Porocephalus crotali Humboldt in Florida. Ohio Jl Sc., XLII, 112.

Brumpt (E.), 1936. - Précis de Parasitologie, II, 1105.

Chabaud (A.-G.) et Chopuet (M.-T.), 1954. - Nymphes du Pentastome Gigliolella (n. gen.) brumpti (Giglioli, 1922) chez un Lémurien. Rev. Parassitologia, XV (4), 331-336, 2 fig.

ChABERT, 1767. - Maladies vermineuses, $2^{\circ}$ édit,, 39-41.

CREPLIN, 1829. - Nouvelles observations d'Entozoaires, 76.

Cú́Not (L.), 1949. - Traité de Zoologie de P.-P. Grassé, VI, 3-75.

Curasson (G.), 1929. - Sur le mode de développement et sur quelques hôtes nouveaux de Porocéphales. Bull. Soc. Path. Exot., XXII, 255-257.

Cuvier (G.), 1817. - Règne animal, $1^{\text {re }}$ édition, IV, 35.

Cuvier (G.), 1830. - Règne animal, $2^{\circ}$ édition.

Devos (R.), 1939. - Deux Porocéphales de crocodiles du Luapula. Ann. Soc. belge Méd. Trop. Bruxelles, XIX, 193-195, 2 pl.

Diesing, 1835. - Monographie du genre Pentastome. Ann. d. Wien. Mus.

Dollfus (R.-Ph.), 1950. - Armillifer L.-W. Sambon, 1922 tombe en synonymie de Nettorhynque H.-D. de Blainville, 1824. Ann. Paras., 130-131.

Dollfus (R.-Ph.) et CANET (J.), 1954. - Sur un Pentastomide, Raillietiella (Heymonsia) hemidactyli M.-L. Hett, 1934, supposé susceptible de parasiter l'homme consécutivement à l'ingestion de lézards vivants. Bull. Soc. Path. Exot., XLVII (3), 401-407.

DUJARDIN (F.), 1845. - Histoire naturelle des Helminthes ou vers intestinaux, Roret édit., Paris, 299-309.

FAIN (A.), 1960. - Diagnoses de deux nouveaux Pentastomidés du Congo belge. Rev. Zool. Bot. Afr., LXI (1-2), 117-118.

FAIN (A.), 1961. - Les Pentastomides de l'Afrique centrale. Musée Royal de l'Afrique centrale, Tervuren, Ann. sér. in $-8^{\circ}$ (Sc. Zool.), $\mathrm{n}^{\circ}$ 92, 1-115.

Fain (A.) et Mortelmans (J.), 1960. - Observations sur le cycle évolutif de Sambonia lohr- 
mani chez le varan. Preuve d'un développement direct chez les Pentastomides. Bull. Acad. Roy. de Belgique (Cl. des Sciences), 5érie, XLVI (6), 518-531, 12 fig.

Fernando (W.), 1953. - Notes on two larval (nymphal) linguatulids from Ceylan. J. Sc., 155-156, pl. 34-35.

FonseCA (F. DE), 1938. - Observaçôes sôbre a ciclo evolutivo de Porocephalus clavatus especialmente sobre o seu tropismo em cobraias. Bol. Biol. São-Paulo, N.S. III, 157-163, 9 fig.

Franchini et Giondano, 1929. - Patologia tropicale. Bologna, La Grafolito.

FRöLICH (J. A.), 1789. - Beschreibung einiger neuer Eingeweidewürmer. Der Naturforscher, II, Halle, XXIV, 148, fig., 11-15, et XXV, 101.

Gedoelst (L.), 1921. - Un Linguatulide nouveau parasite d'un Batracien. Records of the Indian Museum, XXII, part I, 25-26.

Gelormini (N.) et Roveda (R. J.), 1938. - Linguatula serrata. Inst. Paras. Fac. Ag. Veter, Buenos-Aires, I, 3-12, 4 pl.

Giglioli (G.), 1922. - Armillifer brumpti n. sp., nouvelle espèce de Linguatulidé de Madagascar. Bull. Soc. Path. Exot., XV, 565-572.

Giglioli (G.), 1927. - Observations sur la morphologie de l'œuf et de l'embryon chez Porocephalus clavatus (Wyman, 1845, Sambon, 1910). Bull. Soc. Path. Exot., XX, 260-270, 6 fig., pl. IV.

GMELIN (J.-F.), 1800. - Systema naturae, 3069.

Gomez (L.), 1945. - Artroparasitos de la région penibetica. Rev. Ibérico Parasit. Granada, Tom. extraord., 73-105, pl. 1 et 2.

GrÉtillat (S.) et BRYGoo (E.-R.), 1959. - Raillietiella chamaeleonis n. sp., première espèce de Cephalobaenidae (Pentastomida) signalée à Madagascar. Ann. Paras., XXXIV (1-2), 112-120, 12 fig.

HAFFner (V.). 1924. - Die systematische Stellung der Linguatuliden. Zool. Anz., LXI, 209.

Hetr (M. L.), 1915. - On a new species of Pentastomida from a North African Snake (Zamenis ravergieri). Qu. Jl Micro. Sc., LXI (2), 185-200.

HetT (M. L.), 1915. - On some new pentastomids from the Zoological Society Gardens London. Proc. Zool. Soc. London, 115-121.

Hetr (M. L.), 1921. - Notes on a small collection of Pentastomids from the Indian Museum, Calcutta. Rev. Ind. Mus., XXII (3), 163-164.

Hetr (M. L.), 1924. - On the family Linguatulidae. Proceed. Zool. Soc. London, (I), 107159.

HeTt (M. L.), 1924. - Zoological Results of the third Tanganyika Expedition. Report on the Linguatulidae. Proc. Zool. Soc. London, (I), 161.

HetT (M. L.), 1934. - On a collection of Linguatulids from Burma with description of a new subgenus. Proc. Zool. Soc. London, 427-431.

Heymons (R.), 1922. - Beitrag zur Systematik und Morphologie der Zugenwürmer. Zool. Anz., 55.

Heymons (R.), 1926. - Beiträge zur Kenntnis der Gattung Raillietiella Samb. Zool. Anz., XLVII, 45-56.

Heymons (R.), 1932. - Ein neue Pentastomida von den Philippinen. Zool. Anz., XCVII, 295-299.

Heymons (R.), 1932. - Ein Beitrag zur Kenntniss der Pentastomida Australiens und benacharter Gebiete. Zeitschr. f. Parasitenk., IV (3), 409-430.

Heymons (R.), 1935. - Pentastomida. Bronns Klassen u. Ordnungen des Tierreichs, Leipzig, Bd I, 1-268 pp., 35 fig. 
Heymons (R.), 1939. - Beiträge zur Systematik der Pentastomiden. II. Einige Bemerkenswerte Pentastomiden aus Lacertilien. Zeitschr. f. Parasitenk., X (6), 675-690.

Heymons (R.), 1939. - Idem. III. Pentastomiden mit spiralig gekrümmten Köper formen. Zeitschr. f. Parasitenk., VIII, 77-94.

Heymons (R.), 1940. - Ueber Afrikanische Pentastomida mit besonderer Berücksichtigung der neueren Funde ihres Vorkommens im Belgischen Kongo Stadt und in Kamerun. Rev. Zool. Bot. Afric. Bruxelles, XXXIII (2), 201-224, 2 fig.

Heymons (R.), 1940. - Ueber die Lebenweise der in Krokodilen vorkommenden Pentastomida. Sitzungs Ber. Gesellsch. Naturf. Freunde Berlin, 1940-1941, 253-263.

Heymons (R.), 1941. - Beiträge zur Systematik der Pentastomiden. IV. Zur Kenntnis der Sambonidae. Zeitschr. f. Parasitenk., XII, 317-329, 3 fig.

Heymons (R.), 1941. - Idem IV. Die Typenexemplare von Diesingia megastoma. Zeitschr. f. Parasitenk., XII (3), 330-335, 5 fig.

Heymons (R.), 1942. - Idem. VI. Die Arten der Gattung Alofia im Vergleich mi Sebekia. Zeitschr. f. Parasitenk., XII (4), 419-432, 7 fig.

Heymons (R.), 1942. - Der Nasenwurm des Hundes (Linguatula serrata Frölich), seine Wirte und Beziehungen zur Europaïschen Tierwelt, seine Herkunft und pratiktische Bedeutung auf Grund unsere bisheringen Kenntnisse. Zeitschr. f. Parasitenk., XII, 605- , 8 fig.

Heymons (R.), 1943. - Pentastomida. Exploration Parc National Albert, Bruxelles, Mission G. de Witte, XLIII.

Heymons (R.), Vitzthum (H. Gr.), 1938. - Neue und wenig bekannte Pentastomiden aus Amerika. Zoll. Anz., CIX (5-6), 150-158, 6 fig.

Heymons (R.), Vitzthum (H. Gr.), 1936. - Beiträge zur Systematik der Pentastomiden. Zeitschr. f. Parasitenk., VIII, 1-103.

HILl (H. R.). - The occurence of Linguatulids in Pythons. Bull. S. Calif. Acad. Sc., XXXIII, 117-192, 4 pl.

Hill (H. R.), 1948. - Annotated bibliography of the Linguatulida. Bull. S. Acad. Sc., XLVII, 56-73.

Hobmaier (A. et M.), 1940). - On the life cycle of Linguatula rhinaria. Am. Jl Trop. Med. Baltimore, XX, 199-210.

HoYle (W. E.), 1883. - On a new species of Pentastomum (P. protelis) from the mesentary of Proteles cristatus, with account of its anatom. Trans. Roy. Soc. of Edinburg, XXXII, 165-193, pl. XXVII-XXVIII.

Humboldt (A. von), 1799. - Ansicht. d. Natur., $1^{\text {re }}$ édit., 162 et 227.

Humboldt (A. von), 1809. - Rec. Observ. de Zoologie et d'Anatomie comparée, fasc. 5 et 6, $\mathrm{n}^{\circ} 17,298$.

Keegan (H. L.). - Observations on the Pentastomid Kiricephalus coarctatus (Di Sambon, 1910). Trans. Amer. Micr. Soc. Men., LXII, 194-199.

KeEgAN (H. L.), 1943. - Some host records from the parasitological collection of the state University of Iowa. Bull. Brooklyn. Ent. Soc., XXXVIII, 54-57, 2 fig.

Kishida (K.), 1928. - A new Linguatulid Armillifer yoshidai with notes on the Porocephalidae. Annot. Zool. Japon, XI (4), 369-405.

LAMARCK (J. B. DE), 1816. - Histoire naturelle des Animaux sans vertèbre. Edit. III, 174. LARrousse (F.), 1925. - Larve de Linguatulidae parasite de Bufo mauritanicus. Arch. I.P. Tunis, XIV, 101-105.

Leuckart (R.), 1860. - Bau und Entwicklungs geschichte der Pentastomen nach Unter- 
suchungen besonders von Pentastoma taenioides and Pentastoma denticulatum, Leipzig und Heidelberg.

Macalister (A.), 1874. - On two new species of Pentastoma. Proceed. of Roy. Irish Academy. II. Science, 62, pl. II, fig. 11-13; pl. III, fig. 14.

Mahon (J.). - A new species of Raillietiella a pentastomid from the bearded lizard Amphibolurus barbatus (Cuv.). Proc. Zool. Soc. London, CXXIV, 509-516, 1-6.

Marambio (J. A.), 1946. - Contributión al estudio de los metazoos parasitos del perró. Agric. Chile, IV, 59-71.

Monifz (R.), 1896. - Traité de Parasitologie animale et végétale appliqué à la Médecine, 577.

Mouchet (R.), 1914. - Note sur Porocephalus moniliformis. Bull. Soc. Path. Ex., VII, 497-501 et Arch. f. Sch. u. Trop. Hyg., 1913.

Neuman (G.), 1899. - Sur les Porocéphales du chien et de quelques mammifères. Arch. Parasit., II, 356-361.

Neveu-Lemaire (M.), 1938. - Traité d'Entomologie médicale et vétérinaire. Paris, Vigot édit., 153-173.

Nicoli (R. M.), Golvan (Y.), 1959. - Sur une petite collection de Porocephalidae (Pentastomida) en provenance de l'Angola. Première note. Bulletin de la Société de Pathologie exotique, LII, 145-146.

Nicoli (R. M.), Golvan (Y.), 1962. - Pentastomides de l'Angola. Publ. cult. Co. Diam. Ang. Lisboa.

Noc (F.), 1922. - Infestation massive de la genette du Sénégal par des larves de Porocéphales. Bull. Soc. Path. Exot., XIII, 621-631, pl. I-III.

Noc (F.), 1923. - Sur l'embryon acariforme et les stades larvaires des Linguatulides. Bull. Soc. Path. Exot., XIV, 16.

Noc (F.), Curasson (G.), 1920. - Contribution à l'étude de l'évolution biologique de Porocephalus armillatus Wyman. Bull. Soc. Path. Exot., XIII (8), 656-659.

Noc (F.), Giglioli (G.), 1922. - Linguatulids parasitics in Monitors. The new genus Sambonia. Journ. Trop. Med. Hyg., XV, 276-286.

PAPAdakis (A. M.), 1958. - Human infestation with Linguatula serrata. Trans. Roy. Soc. Trop. Med. Hyg., LII (4), 307.

Parodi (S.), Mazza (S.), 1928. - Nota sobre la Linguatula serrata Frölich. $4^{e}$ Reun. Soc. Arg. Pat. Reg. Norte in Bol. Inst. Clin. Buenos-Aires, 642-643, 2 fig.

PenN (G. H.Jr), 1942. - The life history of Porocephalus crotali a parasite of the Louisiana Muskrat. Jl Paras. Urbana, XXVIII, 277-283, 2 fig.

Penn (G. H. Jr), Martin (E. C.), 1941. - The occurence of porocephaliasis in the Louisiana Muskrat. Jl Wildlife Management, V, 13-14.

PInTo (C.), 1938. - Zooparasitos de Interesse medico e veterinario, Rio-de-Janeiro.

Pujattr (D.), 1949-1950. - Intorno alla Raillietiella orientalis Hett. (Pentastomida) e a suo nuovo ospite intermedio. Riv. Biol. Colon., X, 23-26, 1 fig.

Pujatri (D.). - Nesocia bandicota Bechstein (Muridae) ospite intermedio della Linguatula serrata Frölich. Natura Milano, XL, 18-22, 1 fig.

Pullaz (E. M.), 1936. - A note on the occurence of Linguatula serrata Frölich, 1789 in Australia. Austr. Vet. Sc. Sydney, XII, 61-64.

Rodhain (J.), VuYlstoke (C.), 1932. - Contribution à l'étude des Porocéphales des Crocodiles africains. Rev. Zool. Bot. Afr., XXIII, 1-11.

Roveda (R. J.), 1948. - Pentastoma gracile Diesing, 1835. Rev. Fac. Agron. Veter., BuenosAires, XII, 47-50. 
RUDOLPHI, 1808-1809. - Histoire naturelle des Entozoaires, II, 1-440, pl. 12, fig. 8-12.

RUDOLPHI 1819. - Entozoorum synopsis.

SAmbon (L. W.), 1922. - A synopsis of the family Linguatulidae. Jl Trop. Med. Hyg., XXV, 188-206 et 391-428.

Self (J. T.), 1951. - The auxiliary hooks in Porocephalus crotali Humboldt. Trans. Am. Micr. Soc., LXX, 255-261, 1 fig.

SElF (J. T.), KunTZ (R. E.), 1957. - Pentastomids from african Reptiles and Mammals and from Reptiles of Florida Island, British Salomon Islands (South Pacific). The Jl $\mathrm{f}$. Parasit., XLIII (1), 194-200.

Self (J. T.), Mc Murry (F. B.), 1948. - Porocephalus crotali Humboldt (Pentastomida) in Oklahoma. Jl Parasit., XXXIV, 21-23, 4 fig.

Shipley (A. E.), 1898. - An attempt to revise the family Linguatulidae. Arch. Parasit., I, 52-80, fig.

Southwell (T.), 1924. - On a collection of Linguatulids in the Liverpool Sch. of Tropical Medicine. Ann. Trop. Med. Parasit., XVIII, 515-533.

StILES (C. W.), 1891. - Bau und Entwickelungsgeschichte von Pentastomum proboscideum Rud. und Pentastomum subcylindricum Dies. Zeitschr. f. Wissensch. Zoolog., LII, 85-157.

Stiles (C. W.), Hassall (A. SD), 1928. - Key catalogue of parasites reported for Primates, Monkeys and Lemur, 479.

Tubangur (M. A.), 1924. - Two larval parasites from the Philippine palm civet (Paradoxurus philippinensis). Phil. Jl Sc., XXIV, 749-753.

Tubangui (M. A.), Masilungan (V. A.), 1936. - Notes on Philippine Linguatulids (Arthropoda. Pentastomida). Phil. Jl Sc., LX, 399-403, 3 fig.

VAN Beneden (P. J.), 1849. - Recherches sur l'organisation et le développement des Linguatules. Mém. Acad. de Bruxelles, Ann. Sc. Nat., $3^{\circ}$ série.

VANDEL (M.), 1949. - Embranchement des Arthropodes. Généralités. In Grassé P.-P. Traité de Zoologie, VI, 79-158.

VAN DEN BERGHE (L.), 1939. - Observations nouvelles sur le développement des Porocéphales. Trav. St. Zool. Wimereux. Vol. jub. M. Caullery, XIII, 39-45, 1 fig., 1 pl.

Venard (C. E.), Bangham (R. V.), 1941. - Sebekia oxycephala (Pentastomida) from Florida fishes and some notes on the morphology of the larvae. Ohio Jl Sc., XLI, 23-28, $1 \mathrm{pl}$.

Walton (A. C.), 1947. - Parasites of the Ranidae (Amphibia). Jl Parasit., XXXIII, 25-27.

WitTICK (R.-J.), 1943. - Aracknida : Pentastomida in Smart: Handbook identif. of Insects of medic. import. London.

Woodland (W. N.), 1920. - On a remarquable new species of Porocephalus (P. pomeroyi n. sp.) from the fore-gut of a nigeria cobra. Parasit., XII, 337-340.

Woodland (W. N.), 1923. - Porocephalus pomeroyi. Jl Trop. Med. Hyg., XXVI, 45.

WYMAn (J.), 1845. — Proceedings of Boston Society, II, 295.

YUtra (L. M.), 1954. - The occurence of a nymphal Linguatulid (Arthropoda, Pentastomida) in the lungs of the domestic cat. Phil. Jl, LXXXIII, 119-121, 1 pl.

ZEDER, 1803. - Naturgesch, 372.

ZENKER (J. C.), 1827. - Parasitae corporis humani internae seu vermes intestinales hominis, Lipsiae, vIII + 1112 pp.

(Laboratoire de Parasitologie, Faculté de Médecine de Marseille) 